\title{
Effect of Distributed Patch of Smooth Roughness Elements on Transition in a High-Speed Boundary Layer
}

\author{
Meelan Choudhari, ${ }^{*}$ Fei Li, ${ }^{\dagger}$ \\ NASA Langley Research Center, Hampton, VA, 23681 \\ Pedro Paredes: \\ National Institute of Aerospace, Hampton, VA, 23666
}

Surface roughness is known to have a substantial impact on the aerothermodynamic loading of hypersonic vehicles, particularly via its influence on the laminar-turbulent transition process within the boundary layer. Numerical simulations are performed to investigate the effects of a distributed region of densely packed, smooth-shaped roughness elements on the laminar boundary layer over a 7-degree half-angle, circular cone for flow conditions corresponding to a selected trajectory point from the ascent phase of the HIFiRE-1 flight experiment. For peak-to-valley roughness heights of 50 percent or less in comparison with the thickness of the unperturbed boundary layer, the computations converge to a stationary flow, suggesting that the flow is globally stable. Analysis of convective instabilities in the wake of the roughness patch indicates two dominant families of unstable disturbances, namely, a high frequency mode that corresponds to Mack mode waves modified by the wake and a lower frequency mode that corresponds to shear layer instabilities associated with the streaks in the roughness wake. Even though the peak growth rate of the later mode is more than 35 percent greater than the peak growth rates of the Mack modes, the latter modes achieve higher amplification ratios, and hence, are likely to dominate the onset of transition, which is estimated to occur slightly later than that in the unperturbed, i.e., smooth surface boundary layer. Additional computations are performed to investigate the effects of various roughness patch configurations on a Mach 3.5 flat plate boundary layer, to help guide an upcoming experiment in the Mach 3.5 Supersonic Low Disturbance Tunnel at NASA Langley Research Center. In this case, the cumulative reinforcement of basic state distortion over the length of the roughness patch is predicted to yield a significantly earlier transition than that over a smooth plate or a plate with a shorter length roughness patch.

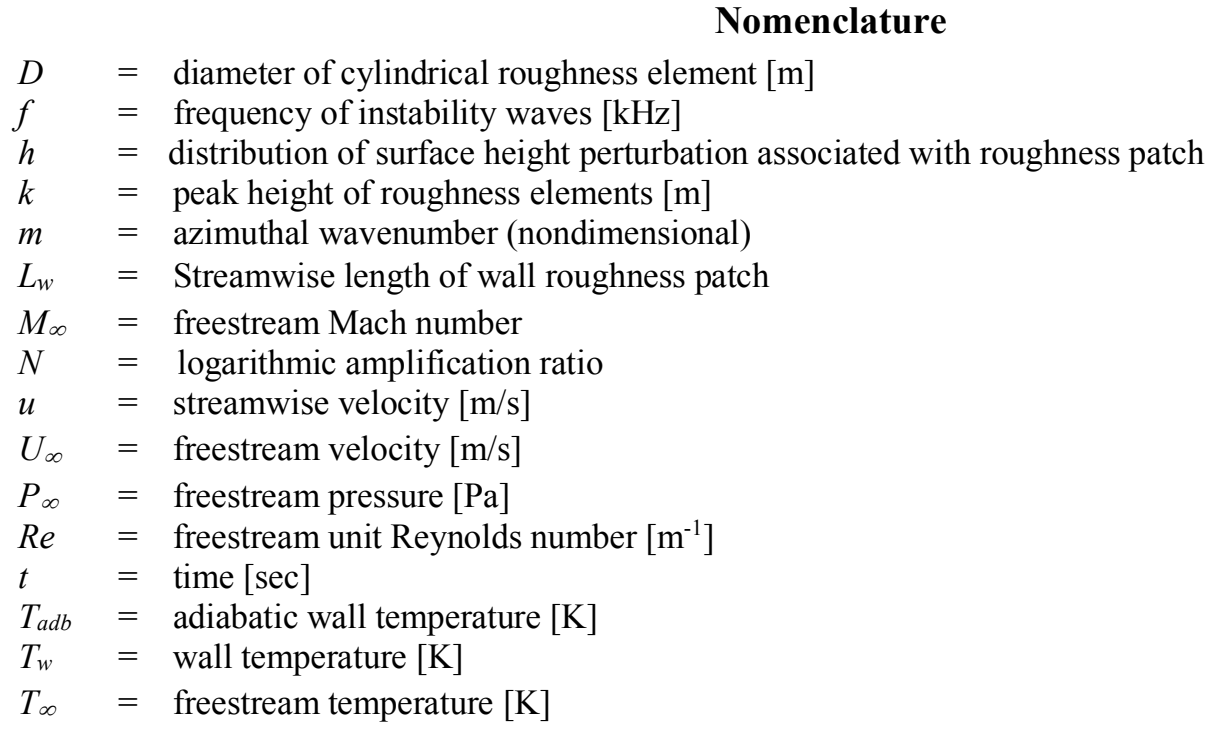

\footnotetext{
*Aerospace Technologist, Meelan.M.Choudhari@nasa.gov, Associate Fellow, AIAA.

† Aerospace Technologist, Fei.Li@nasa.gov

* Research Engineer, pedro.paredes@nasa.gov, Senior Member, AIAA.
} 
$x=$ axial coordinate $[\mathrm{m}]$

$y=$ wall-normal distance $[\mathrm{m}]$

$z=$ azimuthal arc length [m]

$\delta=$ boundary layer thickness [m]

$\lambda_{w}=$ axial wavelength of wall waviness [m]

$\lambda_{\phi} \quad=$ azimuthal wavelength of wall waviness [radians]

$\lambda_{z}=$ spanwise wavelength of wall waviness [m]

$\phi \quad=$ azimuthal angle [radians]

$\sigma=$ spatial amplification rate $\left[\mathrm{m}^{-1}\right]$

\section{Introduction}

Boundary layer transition from the laminar to a turbulent state is known to have a major impact on the design and performance of high-speed flight vehicles. In particular, the thermal protection system (TPS) of the vehicle must be designed to ensure adequate protection for the interior payload. In general, the TPS can be of three types: (i) passive reusable, (ii) active reusable, and (iii) ablative, nonreusable. The ablative TPS is well-suited for reentry vehicles that encounter severe aerothermodynamic environments, particularly when it is not essential to consider reusable TPS. Most planetary entry, descent, and landing (EDL) systems use ablative TPS. The boundary-layer transition characteristics can influence both the surface heating rate (which determines the type of TPS to be employed) and the overall heat load (which controls the thickness of the TPS and, in turn, the associated weight penalty).

When the vehicle surface is relatively smooth, the transition process is initiated by linear instabilities of the laminar boundary layer, including streamwise instabilities in the form of first and second (or Mack) mode instabilities, Görtler instabilities associated with centrifugal effects due to concave curvature of the boundary layer streamlines, and stationary and/or traveling modes of crossflow instability in three-dimensional boundary layers. Transition may also occur right along the leading edge of an aerodynamic surface, either as a result of streamwise instabilities of the attachment line boundary layer or due to a form of "bypass" transition caused by the convection of high-amplitude fluctuations from juncture regions involving turbulent flow over an adjacent surface such as a vehicle fuselage.

In practice, the above-mentioned transition scenarios can be significantly altered by the frequent occurrence of surface roughness over real flight vehicles. Here, the term surface roughness is used in a broad sense to denote all short-scale variations in surface geometry that would invalidate the assumptions of the classical boundary layer theory by introducing rapid spatial gradients in the flowfield adjacent to the surface. The geometry variations may be either local (i.e., discrete, isolated roughness elements) or nonlocal (i.e., distributed) in their spatial extent. Furthermore, the surface height distribution may vary along a single direction along the surface (i.e., 2D roughness) or can be a function of both surface coordinates (i.e., 3D roughness). The magnitude of height perturbations may range from rather small values such that the roughness crests are buried deep within the boundary layer, to rather large magnitudes where the roughness contour extends well beyond the edge of the unperturbed boundary layer. Other significant roughness attributes include its length scales along both crossflow and streamwise directions (with the ratio of these two length scales denoting the planform aspect ratio of representative elements), shape, and orientation with respect to the inviscid streamline. Depending on the uncertainties related to the underlying cause for the surface roughness and/or the geometric complexity of the roughness height distribution, the latter may not always be fully deterministic and, at times, may need to be treated as either partially or fully stochastic in nature.

Examples of discrete roughness elements encountered in practical applications include protuberances or cavities that arise as a byproduct of the structural design of the vehicle or as a result of damage to the heat shield due to debris impact during vehicle launch, and gap fillers protruding in between the tiles of the thermal protection system (TPS). Other examples of surface roughness include: distributed surface imperfections due to spallation or nonuniform surface recession over an ablative heat shield or due to misalignment of steps and gaps between the tiles of a TPS surface; other manufacturing imperfections such as surface waviness, and steps or gaps created during the assembly of different components; or due to differential thermal expansion during the flight, and arrays of discrete roughness elements that are deployed as tripping devices over the forebody of hypersonic vehicles with scramjet propulsion.

In general, the presence of surface roughness can influence the aerothermodynamics of high-speed vehicles in two different ways. If the approaching boundary layer flow is already turbulent, then the presence of surface roughness can augment the heat flux associated with the turbulent flow. On the other hand, when the incoming boundary-layer flow is laminar, the presence of 3D surface roughness tends to accelerate the laminar-turbulent 
transition process. Because boundary layer transition can increase the surface heating rates by a factor of nearly four to eight, roughness effects become very important when they cause the transition front to move close to the leading edge (or the nose) of the vehicle surface. Premature transition on reentry vehicles used for human space flight can jeopardize the structural integrity of the entire vehicle and pose a major safety hazard as shown by the investigations related to the 2005 accident involving the shuttle orbiter Columbia. ${ }^{1}$ While roughness-induced tripping of the boundary layer leads to undesirable consequences in a number of practical applications, artificial roughness elements are often employed in scramjet applications to intentionally trip the boundary layer on the forebody of the vehicle, so as to prevent engine unstart without producing unacceptable levels of flow nonuniformities at the entrance to the combustor inlet.

Empirical observations involving discrete roughness elements ${ }^{2,3}$ have identified the maximum roughness height as the dominant parameter controlling the effects on the laminar-turbulent transition process. Measurements suggest that when the roughness height exceeds a critical value, the transition front begins to move upstream relative to that over a smooth surface in the same disturbance environment. The rate of upstream movement slows down at sufficiently large heights and, for roughness heights larger than the so-called effective trip height, the onset of transition appears to asymptote to a location that may range from just behind the roughness element to a finite distance downstream. Both the critical and effective values of the roughness height parameter are influenced by the shape of the roughness element, and also by the external disturbance environment as demonstrated by the recent experiments in the Mach 6 quiet tunnel at Purdue University. ${ }^{3}$

Despite the common occurrence of surface roughness and its well-known impact on the vehicle design and operation for high-speed flight, the physical mechanisms underlying the transition process behind discrete roughness elements were not understood for a long time. Consequently, predictions in practical applications were based on empirical prediction criteria derived from measurement databases involving limited data from prior flights and supplementary measurements in conventional wind tunnel facilities. The extensive research performed in support of shuttle orbiter Discovery's Return-To-Flight (RTF) led to a first generation, rapid predictive capability for boundary layer transition due to damage or repair to the thermal protection system. ${ }^{1}$ The usefulness of such empirical methodology is, however, limited by the significant uncertainty associated with the correlation and the fact that it cannot be reliably extrapolated beyond the limited underlying database. Often, the vehicle design process is based on the assumption of fully turbulent flow (e.g., the X-34 and Orion Multipurpose Crew Vehicles). Such an overly conservative design approach leads to thicker than necessary TPS and increased total weight or, equivalently, reduced payload fraction.

For overviews of earlier research related to roughness effects on transition including both slender/aerodynamic and blunt body configurations, the reader is referred to the reviews by Schneider. ${ }^{4,5}$ More recent research under the NASA Fundamental Aeronautics Program led to significant breakthroughs in the understanding of physical mechanisms responsible for transition due to discrete roughness elements and has also provided constituents toward a next generation, physics-based prediction tool. With sustained effort, a prediction capability of this type could be incorporated into the aerothermodynamic assessment of a broader class of hypersonic vehicles. An overview of the lessons learned from these research efforts was presented by Choudhari et al. ${ }^{6}$

Naturally occurring surface nonuniformities, such as the roughness shapes induced by surface ablation, tend to be spatially distributed in nature. Thus, one must investigate the effects of spatial proximity between different parts of the roughness distribution. In that regard, the simplest configuration consists of multiple, discrete roughness elements, with the downstream elements located within the wakes of the upstream elements. ${ }^{7,8}$ The most complex case involves densely packed roughness distribution with a potentially heterogeneous mixture of roughness scales, sizes, and orientations. ${ }^{9-12}$ A configuration of intermediate complexity involves smooth-shaped, sinusoidal roughness elements distributed over multiple wavelengths. ${ }^{13}$ Specifically, Muppidi et al. ${ }^{13}$ studied a relatively simple distributed roughness configuration involving a linear superposition of two waves that are inclined at equal but opposite angles with respect to the freestream direction underneath a Mach 2.9 flat plate boundary layer. Even though no unsteady disturbances were introduced in their simulation, the flow developed spontaneous instabilities that led to transition just behind the roughness patch. In the present paper, we report on numerical computations of similar, distributed roughness configurations in the context of both a Mach 5.3 boundary layer over a 7-deg. circular cone (Section II) and a Mach 3.5 flat plate boundary layer (Section III). To examine the roughness effects at representative flight conditions, the flow conditions and surface temperature distribution for the Mach 5.3 configuration are selected to model the $t=21.5$ second trajectory point during the HIFiRE-1 flight experiment. ${ }^{14.15}$ On the other hand, the flow conditions for the Mach 3.5 flat-plate configuration correspond to the experiments by Chou et al. ${ }^{8}$ in the Supersonic Low Disturbance Tunnel at NASA Langley Research Center. In the absence of surface roughness, the Mach 5.3 boundary layer supports strong amplification of second mode (i.e., Mack mode) instability waves so that transition onset occurs near 83 percent of the body length. On the other hand, the Mach 3.5 
boundary layer supports relatively weak amplification of first mode instabilities, and hence, transition is not expected to occur within the length of the plate. Thus, together, these two configurations provide a reasonably broad context for investigating the effects of a smooth-shaped distributed roughness configuration. In both Sections II and III, boundary layer modification due to various selected roughness configurations is described first, and is followed by the results pertaining to the instability characteristics of the wake flow behind the roughness patch. A summary of the results presented in those two sections and concluding remarks are presented in Section IV.

\section{Mach 5.3 Flow over a 7-deg. Half-angle Circular Cone}

We first consider the Mach 5.3 boundary layer over a 7-deg. circular cone from the HIFiRE-1 flight experiment, at freestream conditions corresponding to the $t=21.5$ second trajectory point during the ascent phase of the vehicle. We emphasize that the flight test article did not involve a patch of wavy roughness and that the choice of this flow configuration in the present work is purely to allow an analysis of the effects of a distributed roughness patch at flow conditions that are representative of hypersonic flight at high altitudes.

\section{A. Mean Boundary Layer in the Presence of a Wavy Surface Patch}

The HIFiRE-1 flight experiment involved a 1.1 meter long circular cone with a half angle of 7 degrees and a small nose radius of $2.5 \mathrm{~mm}$. The design angle of attack (AoA) was equal to zero degrees with an estimated inflight uncertainty of just under 1 degree. Based on the analysis of the flight test data, this design goal was met during the ascent portion of the actual flight experiment. A detailed analysis of the boundary layer stability during the ascent phase and a correlation between instability amplification and measured transition locations was presented by $\mathrm{Li}$ et al. ${ }^{15} \mathrm{In}$ this paper, we focus on the flow conditions corresponding to the $t=21.5$ second trajectory location as outlined in Table 1 .

Table 1. Freestream conditions at selected trajectory point during ascent phase.

\begin{tabular}{cccccc}
\hline $\begin{array}{c}\text { Time } \\
(\mathrm{s})\end{array}$ & $\begin{array}{c}P_{\infty} \\
(\text { Pascal })\end{array}$ & $\begin{array}{c}T_{\infty} \\
(\mathrm{K})\end{array}$ & $M_{\infty}$ & $\begin{array}{c}\text { Unit Re } \\
\left(10^{6} / \mathrm{m}\right)\end{array}$ & $\begin{array}{c}\text { Altitude } \\
(\mathrm{km})\end{array}$ \\
\hline 21.5 & 6878.1 & 201.4 & 5.30 & 13.42 & 18.86 \\
\hline
\end{tabular}

We examine the effects of a relatively simple distributed roughness configuration involving a deterministic and smooth surface height variations over a spatially extended region. This configuration is modeled after the roughness geometry considered in the computations of Muppidi et al., ${ }^{13}$ who examined the effects of sinusoidal height variations comprised of a linear superposition of two waves that are inclined at equal but opposite angles with respect to the freestream direction. The surface height perturbation used in their study corresponds to

$$
h(x, z)=k \sin \left(2 \pi x / \lambda_{w}\right) \cos \left(2 \pi z / \lambda_{z}\right),
$$

which may also be viewed as multiple spanwise arrays of smoothly shaped roughness elements that are staggered in the streamwise direction. At the primary flow conditions of $M_{\infty}=2.9, R e=25 \times 10^{6} / \mathrm{m}, k / \delta=0.30$ and $\lambda_{w} / \delta=3.375$ at the beginning of the surface waviness, and $T_{w} / T_{a d}=1$, the computed flow became unsteady without any external forcing. The fundamental wavelength of the surface height distribution had very similar values in the streamwise and spanwise directions, respectively. Computations revealed that both skin friction and surface heat transfer began to rise rather rapidly just past the end of roughness, and reached their respective peak values at a distance of approximately $10 \lambda_{w}$ downstream of the aft end of the roughness strip. Flow above the roughness region was characterized by the presence of both a shear layer with strong velocity gradients at a finite height above the wavy surface and a spanwise periodic array of streamwise vortices that developed below the shear layer. Analysis of the computed data also showed that the spatial variations in the wall-normal velocity gave rise to streamwise vorticity that gained in strength with distance along the roughness strip, and also induced streamwise streaks of alternating high and low velocity. Muppidi et al. ${ }^{13}$ argued that the streamwise vortices were a critical part of the transition mechanism in this case and that it was the interaction between these vortices and the shear layer that initiated the laminar breakdown in the flow.

The roughness configurations considered for the axisymmetric HIFiRE-1 configuration are given by

$$
h(x, \phi)=k \sin \left(2 \pi x / \lambda_{w}\right) \cos (m \phi),
$$

and were selected to have identical wavelengths in both axial and azimuthal directions near the start of the roughness patch. Because the cone expands in the downstream direction and the azimuthal wavenumber is fixed, the azimuthal wavelength expands slightly with $x$ in the present case. The roughness region begins at $x=0.49 \mathrm{~m}$, i.e., approximately near the middle of the cone length, and the wavelength $\lambda_{w}$ of the sinusoidal roughness

4

American Institute of Aeronautics and Astronautics 
distribution is equal to $2.1 \mathrm{~mm}$, corresponding to $\lambda_{w} / \delta \approx 2.6$ where $\delta$ denotes the boundary layer thickness near the beginning of the roughness patch. The present paper considers significantly longer roughness regions than that in Ref. [13], extending over an axial length of $L_{w} / \lambda_{w}=10-20$, compared to $L_{w} / \lambda_{w}=5$ in the previous work. Additionally, to minimize the transients associated with the beginning and the end of the roughness patch, we incorporate smooth tapers on the amplitude envelope of the roughness height distribution at both the upstream and downstream ends of the roughness region. The length of the taper region is chosen to be equal to $2 \lambda_{w}$ for all roughness patches considered in the case of the HIFiRE-1 cone.

The disturbances due to the roughness array are computed by using a high-order DNS solver described by Wu et al. ${ }^{16}$ The inflow to the DNS computations is obtained from the unperturbed boundary layer solution from Li et al. ${ }^{15}$ The working fluid is assumed to be perfect gas (air), and the usual constitutive relations for a Newtonian fluid are used: the viscous stress tensor is linearly related to the rate-of-strain tensor, and the heat flux vector is linearly related to the temperature gradient through Fourier's law. The coefficient of viscosity is computed from Sutherlands's law, and the coefficient of thermal conductivity is computed by assuming a constant Prandtl number $\operatorname{Pr}=0.71$. A detailed description of the governing equations and their numerical solution is given by Wu et al. ${ }^{16}$ The inviscid fluxes from the governing equations are computed using a seventh-order weighted essentially nonoscillatory finite-difference WENO scheme introduced by Jiang and Shu; ${ }^{17}$ the present scheme also allows the use of limiters that have been optimized to reduce the numerical dissipation. Both an absolute limiter on the WENO smoothness measurement and a relative limiter on the total variation are employed simultaneously during the simulation. The viscous fluxes are discretized using a fourth-order central difference scheme and time integration is performed using a third-order low-storage Runge-Kutta scheme. ${ }^{18}$ The numerical code has been previously applied to the simulation of turbulence in hypersonic boundary layers ${ }^{19}$ as well as laminar-turbulent transition due to crossflow instability in swept wing boundary layers. ${ }^{20,21}$ The lessons learned from these simulations were applied to develop the computational grid for stationary crossflow evolution in the present work.

The computational domain begins somewhat upstream of the roughness patch and stretches over a length of nearly $70 \lambda_{w}$. The azimuthal extent of the grid consists of a single wavelength in that direction. Along the wallnormal direction, the computational domain is truncated below the shock, with the boundary conditions at the outer surface imposed on the basis of a separate, full domain computation for the smooth cone. The surface temperature distribution is based on the analysis by Li et al. ${ }^{15}$ pertaining to the flight experiment, corresponding to $T_{w} / T_{a d b} \approx 0.34$ within the region of interest. The grid used for the numerical simulations consists of $6720 \times 128 \times 320$ points in the axial $(x)$, azimuthal $(\phi)$, and wall-normal $(Y)$ directions, respectively. The grid points are spaced uniformly in the azimuthal direction, but are clustered near the wall in the wall-normal direction and also in the vicinity of the roughness patch along the streamwise direction, yielding a resolution of 64 to 128 points over each wavelength in both axial and azimuthal directions. For select cases of interest, the numerical accuracy of the basic state calculation was verified a posteriori by using a coarser grid with significantly lower resolution in all three directions.

The computations show that the stationary flow perturbations over the roughness region are nearly sinusoidal at relatively low roughness heights. However, at a moderately large height of $k / \delta \approx 0.2$, the disturbance field becomes distinctly nonlinear. The formation of the streamwise streaks and their progressive strengthening along the length of the roughness region, as first observed by Muppidi et al., ${ }^{13}$ has also been noted in the present higher Mach number case.

The different parts of Fig. 1 indicate the computed flow features at a peak roughness height of $k / \delta \approx 0.25$, including an isosurface at an infinitesimally positive value of the $u$-velocity component (indicating the boundary of the reversed flow region in the vicinity of the roughness patch), crossplane contours of streamwise velocity at a streamwise interval of unit wavelength, and $u$-velocity contours within a selected $x-z$ plane. 

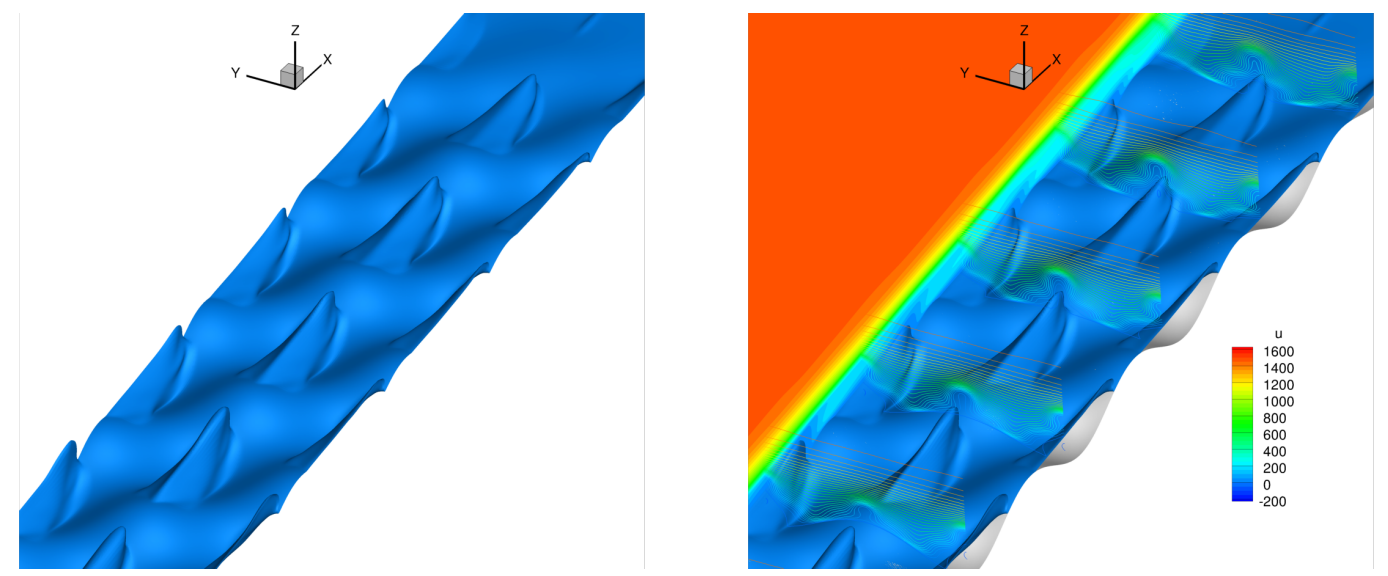

(a) Close-up view of the aft portion of roughness region without (left image) and with (right image) streamwise velocity contours in $x-z$ and $y-z$ planes.

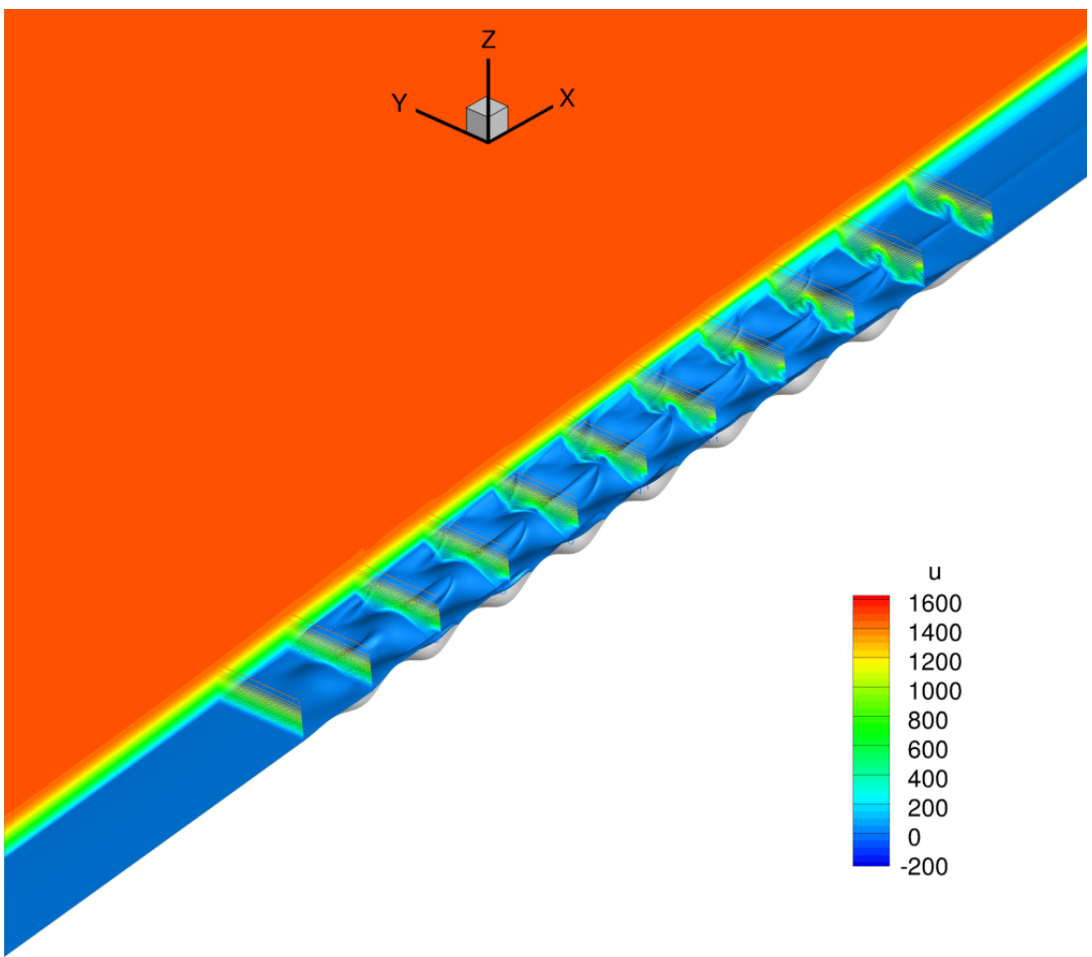

(b) Larger field of view including the entire region of roughness.

Figure 1. Stationary flow above the region of distributed roughness comprised of multiple wavelengths of 3D surface waviness (HIFiRE-1 flight configuration at $t=21.5 \mathrm{sec}$., $k / \delta \approx 0.25$ ). The blue surface indicates the isosurface for a very small positive value of the $u$ velocity component, and the plot also includes crossplane contours of $u$ velocity at a streamwise interval of unit wavelength and $u$-velocity contours within a selected $x-z$ plane.

The isosurface of $u$-velocity component in Fig. 1 indicates a sequence of narrow, finite-length sawtooth like structures (or ridges) that are aligned in the streamwise direction and, hence, cause the higher velocity fluid to go around these ridges, giving rise to the observed streaks in the flow over the rough region. The intensification of streaks over the roughness region, coupled with a decay in streak amplitudes behind the roughness strip, suggests that the boundary layer is likely to support a strong disturbance growth even within the region of waviness, as opposed to mostly in the wake region behind the roughness for the case of discrete, isolated roughness elements. ${ }^{22,23}$ 
Axial evolution of the streak amplitude associated with the mean boundary layer structure from Fig. 1 is plotted in Fig. 2 for three different roughness configurations. These results confirm the highly nonlinear dependence of streak amplitude on the roughness height, similar to the case of an isolated roughness element. A change of peak roughness height from $k / \delta=0.20$ to $k / \delta=0.25$ leads to a significantly larger increase in the streak amplitude levels. A similar increase in streak amplitude occurs as the streamwise extent of the roughness distribution is increased. Specifically, doubling the streamwise extent of the roughness patch at a fixed roughness height of $k / \delta=0.20$ leads to streak amplitudes that are somewhat larger than those induced by a shorter roughness patch with larger roughness height. The results in Fig. 2 also indicate that the decay in streak amplitude behind the roughness region is slower in cases where the initial amplitude is larger.

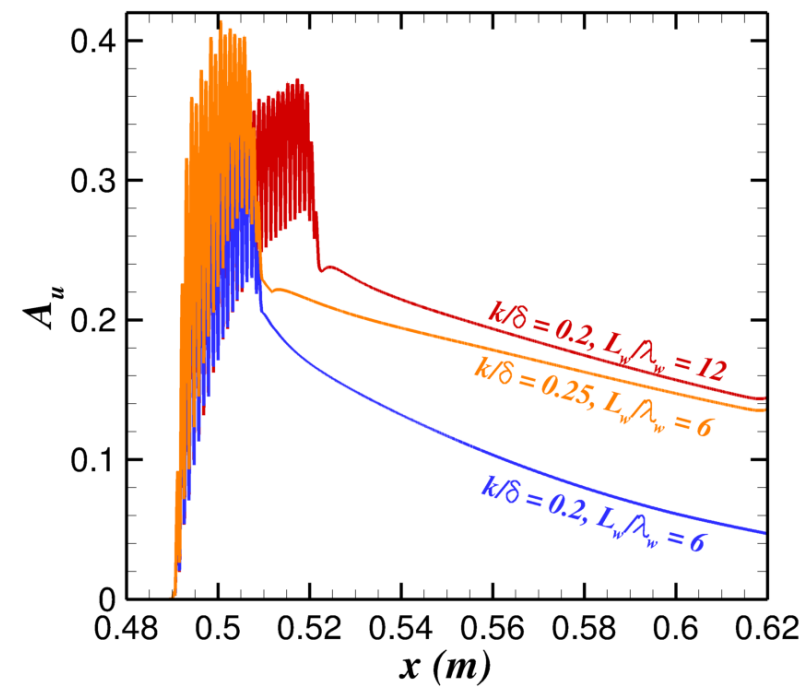

Fig. 2. Streak amplitude evolution for three different roughness configurations with varying roughness height $k / \delta$ or the streamwise extent of wall waviness in terms of number of wavelengths $L_{w} / \lambda_{w}$.

\section{B. Analysis of Instability Amplification in the Presence of Distributed Roughness}

The instability of the boundary layer streaks behind the roughness array was investigated via both a quasiparallel analysis of spatial instability as described by Li and Choudhari ${ }^{24,25}$ and the plane marching PSE as described by Paredes et al. ${ }^{26,27,28}$ Due to the symmetry of the roughness configuration in Eq. (2) across $\phi=0$ rad. and $\phi=\pi / m$ rad., the eigensolutions can be classified on the basis of their symmetry characteristics with respect to those two planes. Accordingly, we introduce the notation of SS, SA, AS, and AA disturbance types on the basis of whether the associated fluctuations in the wall-normal velocity are symmetric (S) or antisymmetric (A) at $\phi=-\pi / m$ rad. and $\phi=0$ rad., respectively. Thus, for example, the SA disturbances are symmetric about the $\phi=-\pi / m \mathrm{rad}$. plane and antisymmetric with respect to the $\phi=0$ rad. plane. The azimuthal wavelength of the SS and AA modes is equal to the fundamental azimuthal wavelength of the roughness distribution (namely, $\lambda_{\phi}=2 \pi / \mathrm{m} \mathrm{rad}$.). In contrast, the other two mode types with mixed symmetry conditions correspond to the first subharmonic with an azimuthal wavelength of $\lambda_{\phi}=4 \pi / \mathrm{m}$ rad., i.e., twice the fundamental wavelength of the roughness patch. Based on the findings of Paredes et al., ${ }^{28}$ instability waves that correspond to higher than the first subharmonic of the roughness wavelength have not been considered in the present analysis.

Similar to the wake of an isolated roughness element, ${ }^{6}$ the wake flow behind the patch of distributed roughness is found to support multiple families of unstable modes. Figure 3(a) displays the predicted variation of the growth rates of selected mode types as a function of the disturbance frequency parameter at multiple stations behind the roughness patch. For comparison, corresponding predictions for the baseline case with no roughness are also shown as dashed curves. The dominant mode of instability in the baseline case corresponds to the second mode instability, i.e., Mack mode disturbances that correspond to trapped acoustic disturbances within a waveguide inside the boundary layer. The peak frequency of these disturbances varies from approximately $600 \mathrm{kHz}$ at $x=0.538 \mathrm{~m}$ (i.e., just behind the downstream of the roughness patch) to about $450 \mathrm{kHz}$ at $x=0.975 \mathrm{~m}$. Basic state distortion by the roughness patch increases the average thickness of the boundary layer, and hence, the peak frequencies of wake instabilitiy modes that arise from the Mack mode instability in the unperturbed boundary layer shift to lower values, namely, approximately $520 \mathrm{kHz}$ at $x=0.538 \mathrm{~m}$ and $430 \mathrm{kHz}$ at $x=0.975 \mathrm{~m}$. A comparison of the range of peak 
frequencies in both cases shows that the frequency bandwidth of the dominant Mack mode instabilities is reduced as a result of the roughness patch. Fig. 3(a) also shows that the peak local growth rates of these high-frequency, Mackmode disturbances also decrease as a result of the basic state distortion induced by the roughness patch. The highest reduction in the peak growth rate is associated with the largest amplitude of wake distortion, i.e., with the region immediately behind the roughness patch. The observed reduction in the peak growth rates of Mack mode disturbances in the presence of the roughness patch agrees qualitatively with the findings of Paredes et al., ${ }^{28}$ who examined the interactions between Mack mode instabilities and optimal growth streaks over the HIFiRE-1 cone at the same flow conditions. Their findings indicated that optimal growth streaks with similar wavelengths can substantially reduce the growth rates of the Mack modes, resulting in a strong delay in transition onset in the presence of those streaks.

Besides the high-frequency instability associated with the Mack modes, the wake of the roughness patch also supports additional unstable modes at lower frequencies, which did not exist in the baseline case. The dominant low-frequency mode plotted in Fig. 3(a), i.e., the AA mode, is centered at approximately $120 \mathrm{kHz}$, i.e., nearly four to five times lower than the peak frequencies of the wake instabilities related to Mack mode instability. As a result of the cold-wall thermal boundary condition at the cone surface, the baseline flow does not support any first mode instability. Hence, the lower frequency instability modes are attributed to the shear-layer instabilities associated with boundary layer streaks induced by the roughness patch. Since the streak amplitudes decrease with distance along the roughness wake, the peak growth rate of these instabilities decreases rather rapidly with increasing $x$. Yet, because the most unstable frequency from this family of unstable modes remains in the vicinity of $120 \mathrm{kHz}$ throughout the wake and the highest local growth rate corresponds to a disturbance from this family (Fig. 3(a)), these modes are able to achieve a peak $\mathrm{N}$-factor of approximately 7 near $x=0.8 \mathrm{~m}$ (Fig. 3(b)). On the other hand, the Mack mode instabilities can continue to amplify past that location, especially as the stabilizing influence of the streaks becomes weaker. Thus, the $\mathrm{N}$-factor envelope for the Mack mode instabilities crosses over the envelope of the shear layer modes near $\mathrm{N}=6$ and, from there on, the Mack-mode instabilities control the $\mathrm{N}$-factor envelope associated with the overall amplification of instability waves within the wake of the roughness patch (Fig. 3(b)).

Fig. 3(b) also shows that the peak $\mathrm{N}$-factor for the Mack mode instabilities behind the roughness patch eventually exceeds the corresponding envelope for the baseline case. However, the crossover does not occur until the $\mathrm{N}$-factor approaches a value of nearly 16 . Thus, if one assumes that the correlation parameter of $\mathrm{N}=14.7$ from the analysis of in-flight transition measurement in the baseline case ${ }^{29,30}$ also applies to transition due to Mack mode instabilities in the presence of the streaks, then the $\mathrm{N}$-factor envelope for the flow behind the roughness patch is still slightly below the envelope for the smooth configuration from the HIFiRE-1 flight experiment. Thus, the present roughness patch would be expected to yield a small delay in transition with respect to the baseline case, unless the shear-layer modes can lead to transition at an $\mathrm{N}$-factor of below 7. The extent of the estimated delay in transition due to Mack mode instabilities is significantly smaller than that observed by Paredes et al., ${ }^{28}$ who found transition delays in excess of 50 percent and even approaching 100 percent of the laminar flow length in the baseline case. This discrepancy in the magntidue of transition delay may be caused by the differences in wall-normal profiles of the boundary layer distortions associated with the streaks in these two studies. Another difference between the optimal growth streaks of Paredes et al. ${ }^{28}$ and the wake of the roughness patch considered in the present paper is related to the amplitude evolution of the stationary streak amplitude. For the transient growth streaks, the streak amplitude first increases to its peak value before undergoing a slow decay. On the other hand, the wake behind the roughness patch begins with the highest amplitude of the streak and decays thereafter within the downstream region. Further work is necessary to consider the various factors contributing to the above discrepancy and to determine which of those factors are likely to dominate the overall effect of boundary layer streaks on the onset of transition. 


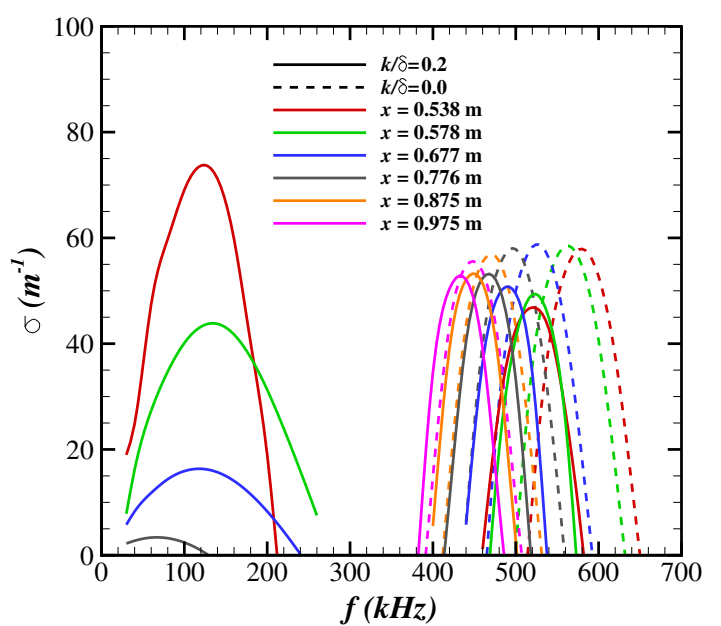

(a) Growth rate variation with frequency for dominant instability modes of $\mathrm{AA}$ and MM families at selected axial locations

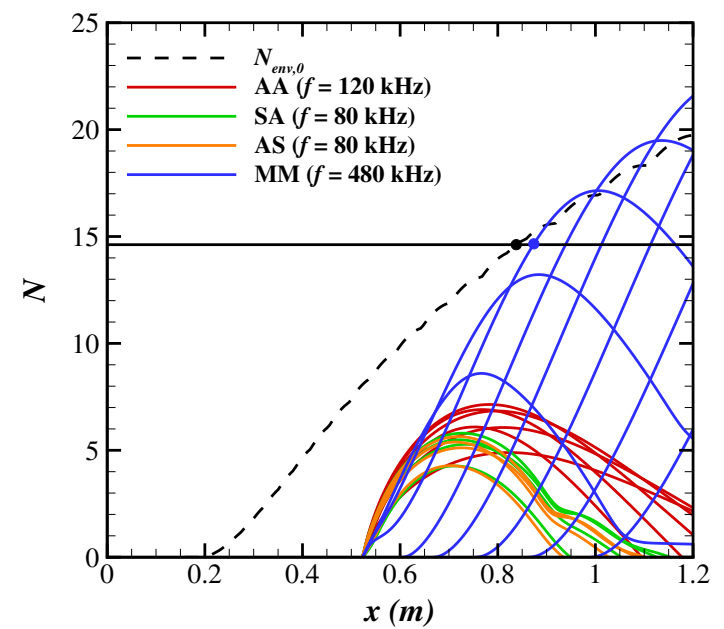

(b) $\mathrm{N}$-factor evolution for selected fixed-frequency disturbances from each mode type. Dashed line denotes $\mathrm{N}$-factor envelope for the baseline case. The black horizontal line along $N=14.7$ indicates transition correlation for the baseline case with no roughness patch.

Figure 3. Instability characteristics for roughness patch with $k / \delta=0.20$ and $L_{w} / \lambda_{w}=12$.

Representative mode shapes for the streamwise velocity fluctuations associated with each of the two dominant families of unstable modes from Fig. 3(a) are shown in Figs. 4(a) and 4(b), respectively. For each family of modes, we indicate the mode shape variation along the length of the wake for a fixed frequency disturbance that approximately corresponds to the disturbance that is likely to be the most relevant to the transition process. For the family of disturbances related to the Mack modes, this disturbance corresponds to $f=480 \mathrm{kHz}$, because it correlates with the measured onset of transition in the baseline case. For the shear layer modes associated with streak instabilities, the selected disturbance corresponds to the most amplified disturbance from that family, i.e., the disturbance with $f=120 \mathrm{kHz}$. The two sets of mode shapes in Figs. 4(a) and 4(b) reveal interesting differences with respect to wall-normal and spanwise locations of peak fluctuations, extent of concentration within the cross-section of the streak, as well as trends in the axial evolution. In the wall-normal direction, the peak $\left|u^{\prime}\right|$ fluctuations associated with the high-frequency instability mode originating from the Mack mode (or acoustic mode) instability are concentrated relatively close to the surface (Fig. 4(a)). In contrast, Fig. 4(b) indicates that the peak velocity fluctuations associated with the lower frequency, intrinsic streak instabilities occur farther away from the surface. In regard to the spanwise locations of peak $\left|u^{\prime}\right|$ fluctuations, for $x \leq 0.823 \mathrm{~m}$, the peak fluctuations induced by the $f=$ $120 \mathrm{kHz}$ mode occur in pairs of localized regions of high spanwise basic state shear that straddle the spanwise crests in boundary layer thickness at a given station. With increasing value of $x$ within this range (accompanied by decreasing streak amplitude), these pairs of peaks progressively shift toward the troughs in spanwise distribution of boundary layer thickness, eventually merging to form a single peak near the trough at $x=0.909 \mathrm{~m}$. In contrast, the peak velocity fluctuations of the MM mode at $f=480 \mathrm{kHz}$ are located beneath each crest location. Initially, the peak $\left|u^{\prime}\right|$ fluctuations underneath the crest at $\phi=0$ rad. (i.e., at $\phi= \pm 2 j \pi / m$ rad. in general, with $j$ being an integer) are higher than the peaks at $\phi= \pm \pi / m$ rad. (i.e., at $\phi= \pm(2 j+1) \pi / m$ rad. in general). However, the former peak(s) become weaker with increasing $\mathrm{x}$, so that the the peaks at $\phi= \pm \pi / m$ rad. become dominant within the aft set of locations shown in Fig. 4(a). The figure also indicates that the peak at $\phi=0$ rad. begins to strengthen again across the last couple of stations; however, the physical reasons behind this trend remain unknown at this stage.

Finally, we also note that the mode shapes of lower frequency, shear-layer instabilities in Fig. 4(b) are relatively more localized within the cross-section of the streaks in comparison with those of the Mack modes from Fig. 4(a). In particular, the fluctuations associated with sinuous shear layer modes are concentrated in the region of higher azimuthal shear $(d u / d \phi)$. By virtue of the odd parity of the AA modes, the streamwise and wall-normal velocity fluctuations associated with these modes are identically zero at the crest of the structure induced by the upwelling. In contrast, because the MM mode corresponds to a trapped acoustic mode, its peak velocity fluctuations do not 
coincide with regions of high mean shear. Furthermore, the Mack mode corresponds to a mode of the SS type, and hence, will lead to varicose oscillations of the streaks, whereas the antisymmetric shear layer mode is of the AA type, and therefore, will induce sinuous oscillations within the wake.

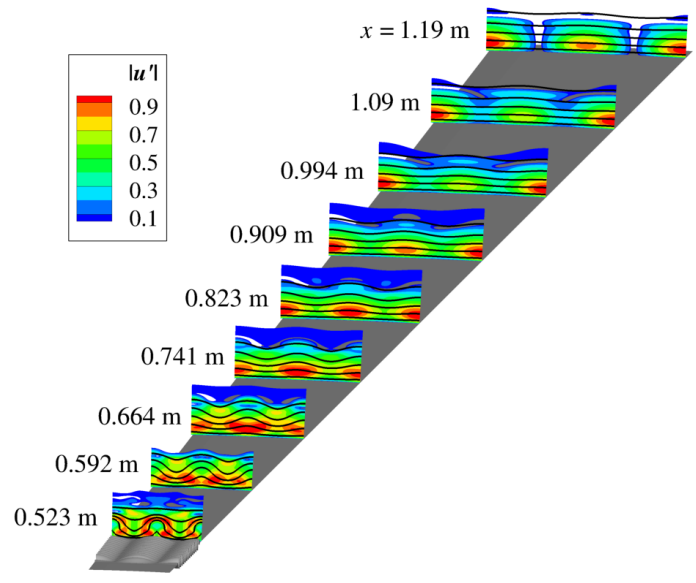

(a) Mack mode (mode MM) with $f=480 \mathrm{kHz}$

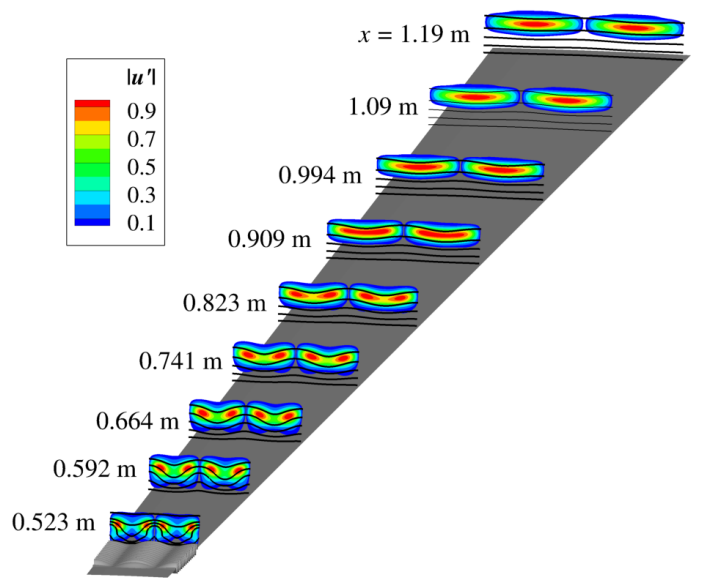

(b) Shear-layer mode (mode AA) with $f=120 \mathrm{kHz}$

Figure 4. Axial evolution of $\left|u^{\prime}\right|$ mode shapes for dominant instability mode from each of the two families of modes in Fig. 3(a) for a roughness patch with $k / \delta=0.20$ and $L_{w} / \lambda_{w}=12$. The azimuthal region plotted in each figure corresponds to $\phi \in(-\pi / m, \pi / m)$ rad. with $m=100$ and the peak fluctuation magnitude at every station is normalized to unity.

The results described in this section have shown that an extended patch of surface roughness comprised of a regular pattern of distributed, smooth-shaped roughness elements (or, equivalently, surface waviness) can induce very similar streamwise streaks as those due to a single array of discrete roughness elements. Furthermore, a spanwise alignment of the protuberance elements of the roughness patch can result in a substantial reinforcement of the streak amplitudes associated with localized segments of the overall distribution, similar to the previous findings for discrete roughness elements arranged in tandem. ${ }^{7,8}$ In this case, increasing the axial length of the patch leads to higher streak amplitudes immediately behind the patch and, in certain cases, even a slower decay in streak amplitudes in comparison with the decay observed behind a shorter segment of the roughness distribution (recall Fig. 2). Somewhat surprisingly, however, for the hypersonic flow configuration examined in this section, a large patch of roughness corresponding to $L_{w} / \lambda_{w}=12\left(L_{w} / \delta \approx 50\right)$ and $k / \delta=0.2$ yields a relatively small change in the Nfactor envelope, in spite of the strong distortion of the boundary layer flow due to the roughness patch. Of course, the parameter space associated with the wavy patch is rather large $\left(\lambda_{w} / \delta, \lambda_{z} / \delta, k / \delta, L_{w} / \lambda_{w}\right.$ and the location of roughness patch) and, quite possibly, variations in other parameters that were not investigated herein could yield a larger effect on the overall disturbance amplification, and hence, on the transition location. A limited parameter study along these lines has been carried out for a Mach 3.5 flat plate boundary layer, a configuration that forms the focus of the following section.

\section{Mach 3.5 Flat Plate Boundary Layer}

In this section, we extend the computations in the previous section to a Mach 3.5 flat plate boundary layer at flow conditions that are relevant to a future experiment in the Supersonic Low Disturbance Tunnel (SLDT) at the NASA Langley Research Center. The nominal freestream conditions selected for the present computations are close to the flow condition from the previous measurements by Chou et al. ${ }^{8}$ namely, $P_{\infty}=2719.9 \mathrm{~Pa}, T_{\infty}=92.55 \mathrm{~K}$, with a unit Reynolds number of $10.77 \times 10^{6}$ per meter. The model surface temperature is assumed to be uniform along the length of the plate and equal to $T_{w}=290 \mathrm{~K}$.

\section{A. Mean Boundary Layer in the Presence of a Patch of Wavy Surface}

Chou et al. ${ }^{8}$ previously reported roughness wake measurements at similar flow conditions for isolated roughness elements. They found that a cylindrical roughness element with a diameter of $D=3.58 \mathrm{~mm}$ and height $k=140 \mu \mathrm{m}$ at a distance of $41.5 \mathrm{~mm}$ downstream from the leading edge led to the amplification of an antisymmetric instability 
mode within the wake region; however, that amplification was insufficient to produce transition within the measurement region. As the roughness elements were increased to $k=280 \mu \mathrm{m}$ (i.e., $k / \delta \approx 0.4$ ), a symmetric instability mode amplified more rapidly in the wake region, resulting in the onset of transition within the length of the plate. For multiple roughness elements aligned in the streamwise direction, an interelement spacing of $4 D$ yielded substantially stronger mean wake distortion in comparison with a single roughness element. However, a roughness element spacing of $2 D$ was found to be too small to yield an effective constructive interfrerence between the wake disturbances induced by the roughness elements in tandem.

For the flat plate configuration, the roughness height distribution across the wavy patch is given by Eq. (1). As indicated in Table 1, a majority of the computations in the present work are carried out for the case where $\lambda_{w}=\lambda_{z}=$ $6.25 \mathrm{~mm}$, which corresponds to a half wavelength (i.e., protuberance portion within a single wavelength) of 3.25 $\mathrm{mm}$. While the half wavelength of a sinusoidal distribution is not exactly equivalent to the diameter of a discrete, cylindrical roughness element, it may be seen that the half wavelength in the present case is comparable to the roughness diameter from the previous SLDT experiment for cylindrical roughness elements. In all cases, the roughness patch nominally begins near $x=0.0288 \mathrm{~m}$ (where the local boundary layer thickness is slightly less than $0.6 \mathrm{~mm}$ ), with the patch length ranging from $L_{w} / \lambda_{w}=0.5$ to $L_{w} / \lambda_{w}=15.0$ and the roughness height from $k=72 \mu \mathrm{m}$ up to $k=340 \mu \mathrm{m}$. For the longer roughness patches of interest in this case, the length of the roughness patch is comparable to the distance between the plate leading edge and the beginning of the patch. Therefore, the thickness of the unperturbed boundary layer varies more than 35 percent over the length of the patch, and for that reason, we choose to characterize the patch height in absolute units, rather than as the nondimensional parameter $k / \delta$, as in the previous section. The flat plate computations covered streamwise wavelengths from $\lambda_{w}=1.7 \mathrm{~mm}$ to $\lambda_{w}=6.25 \mathrm{~mm}$, along with $\lambda_{z}=1.7 \mathrm{~mm}$ and $\lambda_{z}=6.25 \mathrm{~mm}$. A comprehensive analysis of the various cases is still ongoing, and therefore, the present section will focus on a small number of selected cases from Table 1 .

Table 1 Roughness patches investigated for Mach 3.5 flat plate

\begin{tabular}{ccccc}
\hline \hline$k, \mu \mathrm{m}$ & $\lambda_{w}, \mathrm{~mm}$ & $\lambda_{z}, \mathrm{~mm}$ & $L_{w} / \lambda_{w}$ & Remark \\
\hline 272 & 6.25 & 6.25 & $0.5,1.0,2.0,4.0,8.0$ & N/A \\
340 & 6.25 & 6.25 & $0.5,1.0,2.0,4.0,8.0,12.0$ & N/A \\
136,272 & 1.7 & 6.25 & 15.0 & N/A \\
$68,136,204,272$ & 6.25 & 1.7 & 4.0 & N/A \\
272 & 4.2 & 4.2 & 6.0 & N/A \\
272 & 3.125 & 3.125 & 8.0 & N/A \\
$68,102,136,204,272$ & 1.7 & 1.7 & 15.0 & $\begin{array}{c}\text { Protuberances only, } \\
\text { Dimples only } \\
272,340\end{array}$ \\
272 & 6.25 & 6.25 & 0.5 & $\begin{array}{c}\text { Protuberances only, } \\
\text { Dimples only }\end{array}$ \\
\hline \hline
\end{tabular}

In the interest of enabling a more efficient parameter study that will provide guidance for a future experiment involving a patch of surface waviness, a production CFD code in the form of the VULCAN (Viscous Upwind aLgorithm for Complex flow ANalysis ${ }^{29}$ software was used in lieu of the higher order DNS code to compute the basic state flow distortion due to the patch of distributed surface roughness. The VULCAN code solves the unsteady, conservation equations appropriate for laminar or turbulent flow of calorically or thermally perfect gases with a spatially second-order accurate cell-centered finite-volume scheme. In the present computations, the inviscid fluxes were constructed using the MUSCL $\kappa=0$ scheme, the van Albada gradient $\operatorname{limiter}^{30}$ and the Low Dissipation Flux Split Scheme (LDFSS) of Edwards. ${ }^{31,32}$ The cell face gradients required to construct the viscous fluxes were obtained using an auxiliary control volume approach that results in a compact viscous stencil that produces a second-order accurate approximation of the full Navier-Stokes viscous fluxes. A typical grid used in the computations described herein involved $6720 \times 120 \times 720$ points in the streamwise, spanwise, and wall-normal directions, respectively. Additional computations were performed with grids that were coarser by a factor of 1.5 in each direction to ensure that the computed flow solutions showed rather minor changes in the predicted wake distortions.

Qualitative features of the streamwise evolution in the stationary wake of a roughness patch over the Mach 3.5 flat plate are analogous to those described in Section II for the HIFiRE-1 cone. For the purpose of illustration, Mach 
number contours at selected stations for the case of $\lambda_{w}=6.25 \mathrm{~mm}, L_{w} / \lambda_{w}=4.0, k=272 \mu \mathrm{m}$ are shown in Fig. 5. Figure 5(a) reveals the flow evolution within the region of the roughness patch. Mach number contours near the centerplane $(z=0)$ indicate the cyclic pattern of flow acceleration over the rising segments of the surface topography and retardation over the lee side of those segments. Concomittantly, one observes a progressive build up in the size of the blue shaded region within the troughs of successive wavelengths of the roughness patch, which gives rise to increased boundary layer thickness along the symmetry planes at $z / \lambda_{z}= \pm 0.5 j$, with $j$ being an arbitrary integer. The spanwise undulations in streamwise velocity distribution are sustained in the wake region behind the roughness patch, giving rise to similar streaks as those observed behind a single array of roughness elements. ${ }^{33}$

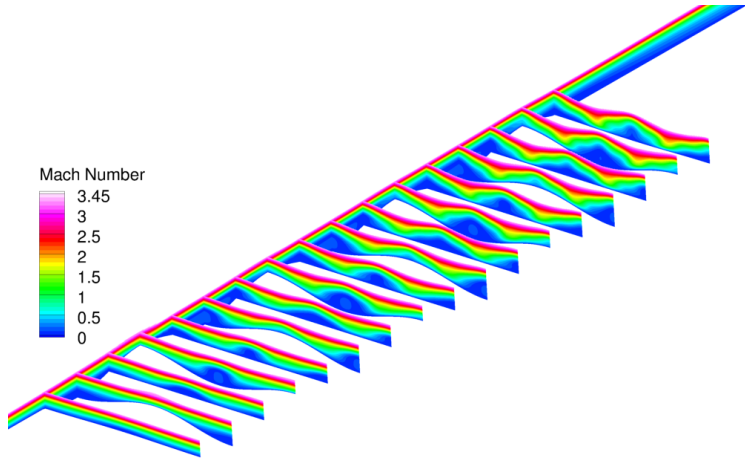

(a) Nearfield contours within the region of the roughness patch at streamwise intervals of $\lambda_{w} / 4$

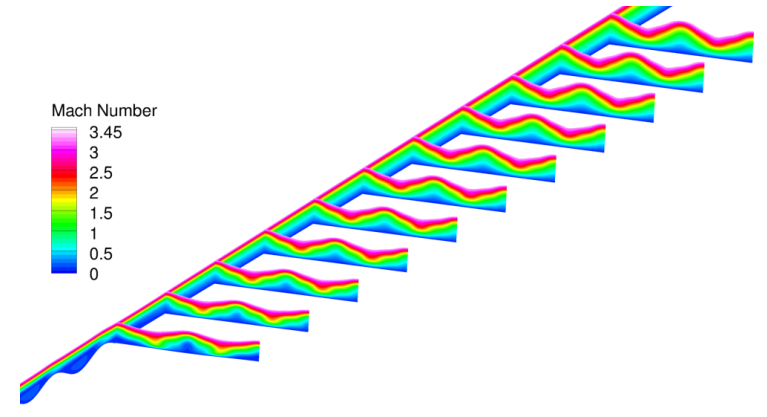

(b) Wake contours behind the roughness patch at streamwise intervals of $\lambda_{w}$

Figure 5. Mach number contours at selected stations above and behind the roughness patch with $\lambda_{w}=6.25$ $\mathrm{mm}, k=272 \mu \mathrm{m}$, and $L_{w} / \lambda_{w}=4.0$. The contours at each streamwise station cover a spanwise interval of $z / \lambda_{z}=[-0.5,0.5]$. For reference, the streamwise evolution of flow within the $z_{z} / \lambda_{z}=-0.5$ plane is also shown along the left boundary of the plot.

To highlight the effects of the length $L_{w}$ of the roughness patch as well as of the proximity between protuberances and dimples associated with the roughness patches considered herein, Fig. 6 shows a comparison between Mach number contours for several roughness configurations at three selected stations, namely, $x=0.06 \mathrm{~m}$, $0.12 \mathrm{~m}$, and $0.30 \mathrm{~m}$, respectively. The streamwise wavelength of the roughness patch is held fixed at $\lambda_{w}=6.25 \mathrm{~mm}$ and the most upstream station at $x=0.06 \mathrm{~m}$ lies approximately one wavelength beyond the trailing edge of the roughness patch with $L_{w} / \lambda_{w}=4.0$. Figures 6(a) through 6(c) display the Mach number contours for the shortest roughness patches considered in this paper, namely, those with $L_{w} / \lambda_{w}=0.5$. The localized distribution of roughness height associated with an $L_{w} / \lambda_{w}=0.5$ roughness patch may be viewed as a single, spanwise periodic array of roughness elements with alternating protuberances and dimples. A comparison between the Mach number contours from Figs. 6(a) and 6(b) reveals the considerable strengthening of the streaks when the roughness height parameter $k$ is increased by only 25 percent, highlighting the nonlinear dependence of the streak amplitude on $k$. Fig. 6(c) displays the Mach number contours associated with just the protuberance segments of the array from Fig. 6(a). A comparison between Figs. 6(a) and 6(c) indicates that the surface dimples associated with the sinusoidal spanwise distribution of roughness height have a lesser effect on the streaks in comparison with the surface protuberances.

The effect of roughness patch length on the streaks within the roughness patch wake may be inferred by comparing the streak evolution in Fig. 6(a) with Figs. 6(d) and 6(e). Roughness height distribution for the $L_{w} / \lambda_{w}=$ 1.0 case in Fig. 6(d) may be viewed as a superposition of two identical patches with $L_{w} / \lambda_{w}=0.5$ that are out of phase with respect to each other, i.e., entail a spatial shift equal to one half wavelength in both streamwise and spanwise directions. Thus, the Mach number contours for this case illustrate the effects of adding dimples behind protuberances and vice versa. Visually, the streak amplitude in the $L_{w} / \lambda_{w}=1.0$ case appears to be similar to that for the $L_{w} / \lambda_{w}=0.5$ case, with the primary difference between the two involving additional modulation of the contours in the $L_{w} / \lambda_{w}=1.0$ case. On the other hand, although not shown, the wake amplitude at $x=0.06 \mathrm{~m}$ for the $L_{w} / \lambda_{w}=4.0$ case (Fig. 6(e)) is nearly twice as large as that for the localized patch with $L_{w} / \lambda_{w}=0.5$.

12

American Institute of Aeronautics and Astronautics 

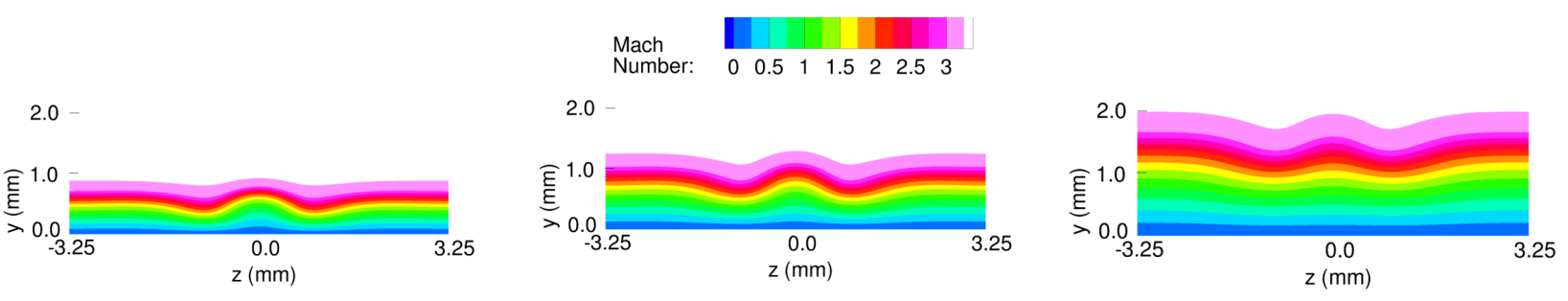

(a) $L_{w} / \lambda_{w}=0.5$
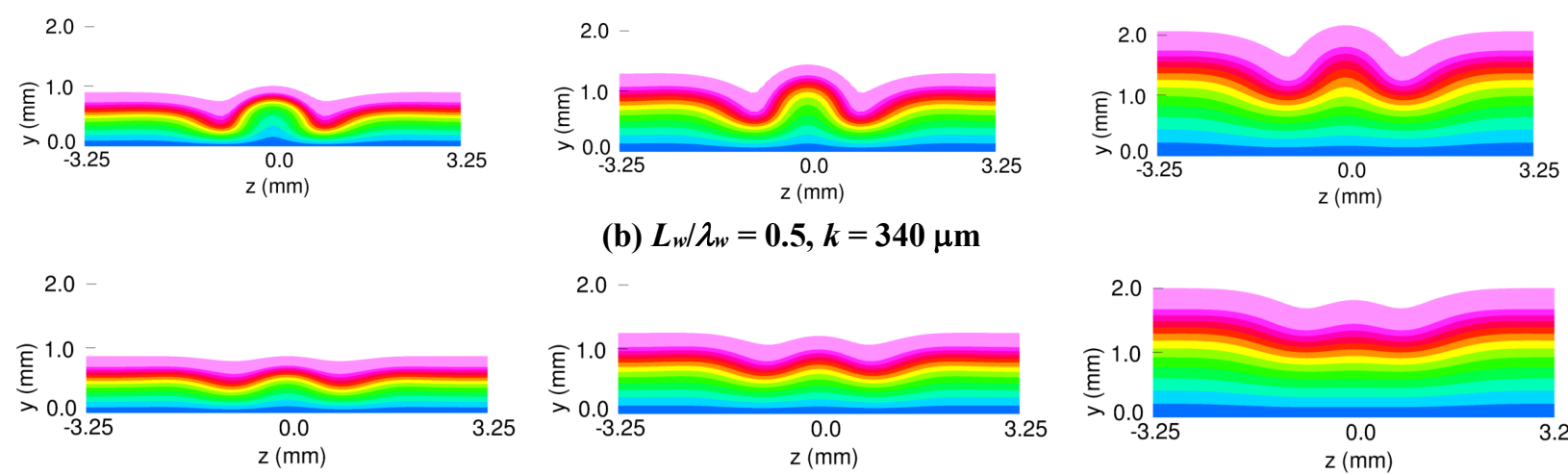

(b) $L_{w} / \lambda_{w}=0.5, k=340 \mu \mathrm{m}$
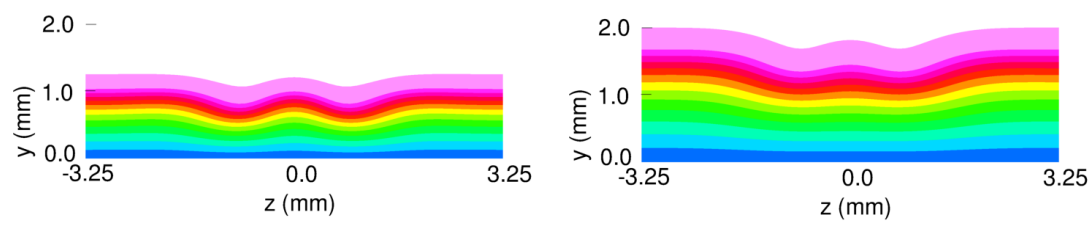

(c) $L_{w} / \lambda_{w}=0.5$, protuberances only
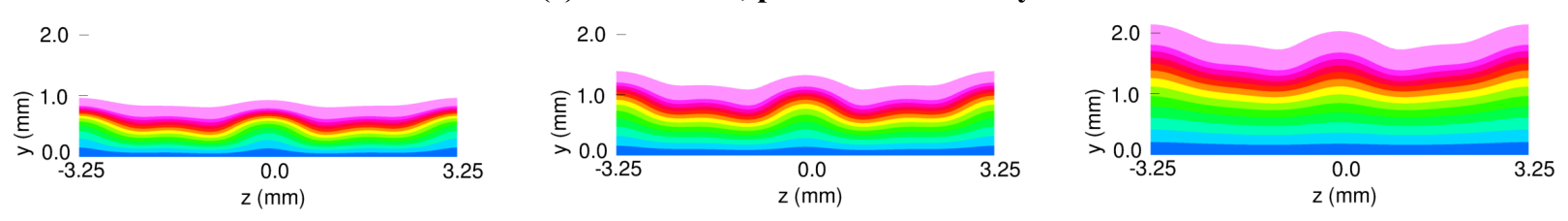

(d) $L_{w} / \lambda_{w}=1.0$
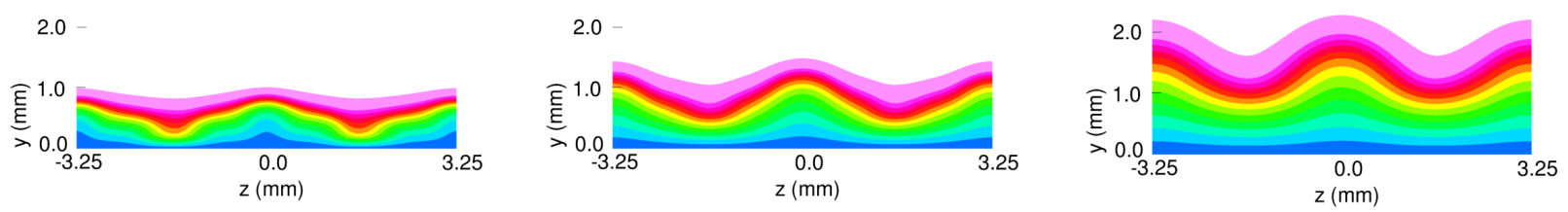

(e) $L_{w} / \lambda_{w}=4.0$
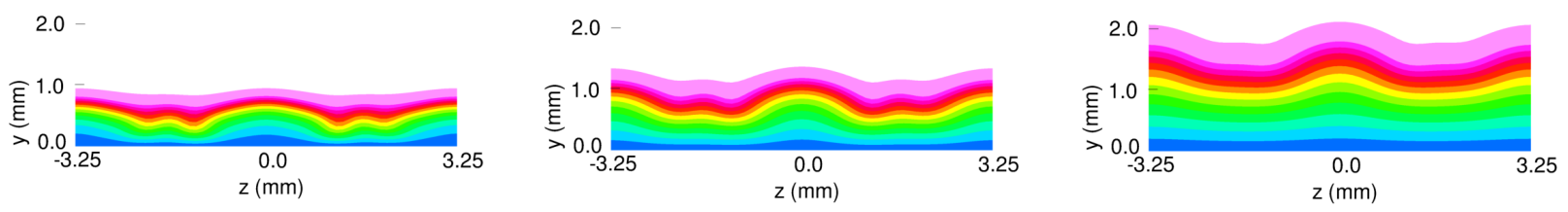

(f) $L_{w} / \lambda_{w}=4.0$, protuberances only
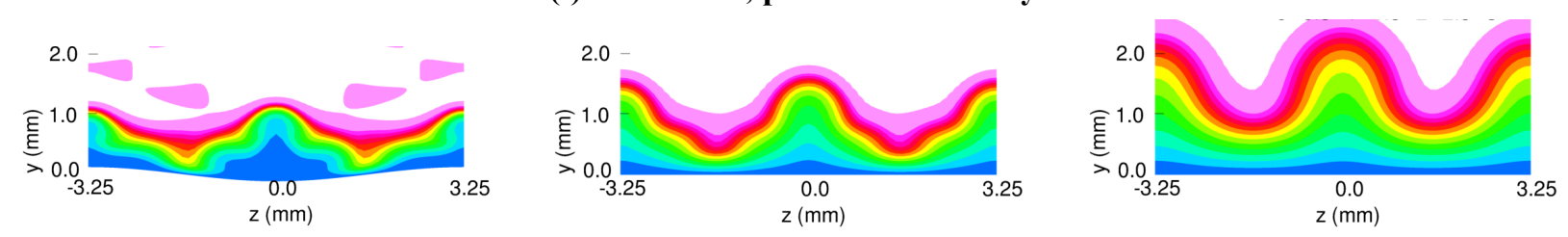

(g) $L_{w} / \lambda_{w}=8.0, k=340 \mu \mathrm{m}$

Figure 6. Comparison of Mach number contours at selected stations within the wake of selected roughness patches. Unless specified otherwise, each patch has a streamwise wavelength of $\lambda_{w}=6.25 \mathrm{~mm}$ and a roughness height of $k=272 \mu \mathrm{m}$. The selected stations, from left to right, correspond to $x=0.06$ $\mathrm{m}, 0.12 \mathrm{~m}$, and $0.30 \mathrm{~m}$, respectively. The legend from Fig. 6(a) applies to all parts of the figure. 
Comparison between Figs. 6(e) and 6(f) illustrates how the inclusion of surface dimples in the former case leads to stronger streaks in the far wake region. However, this increase in streak amplitude is not as strong as the anticipated increase from a doublingof the protuberance height. This suggests that the peak to valley height of a homogeneous patch of waviness is not quite equivalent to the peak height in a protuberance only roughness distribution. Finally, for roughness patches with a larger height parameter corresponding to $k=340 \mu \mathrm{m}$, a comparison of the Mach number contours from Figs. 6(b) and 6(g) again confirms the large increase in streak amplitudes enabled by a longer roughness patch.

\section{B. Modal Instability Amplification behind the Roughness Patch}

Wake instability analysis similar to that of the HIFiRE-1 configuration in section II.A was performed for the $L_{w} / \lambda_{w}=0.5$ and $L_{w} / \lambda_{w}=4.0$ roughness patches from Figs. 6(a) and 6(e), respectively. Both cases correspond to $\lambda_{w}=$ $\lambda_{z}=6.25 \mathrm{~mm}$ and $k=272 \mu \mathrm{m}$. Streamwise evolution of $\mathrm{N}$-factors for dominant instabilitiy modes corresponding to each set of spanwise boundary conditions (i.e., mode types SS, AA, SA, and AS, respectively) is plotted in Fig. 7(a) for the roughness patch with $L_{w} / \lambda_{w}=0.5$ and in Fig. 7(b) for $L_{w} / \lambda_{w}=4.0$.

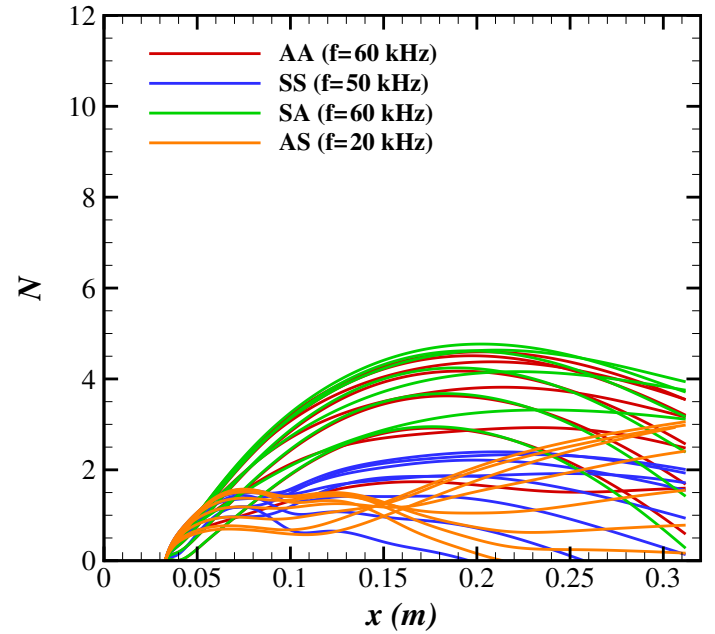

(a) $L_{w} / \lambda_{w}=0.5$

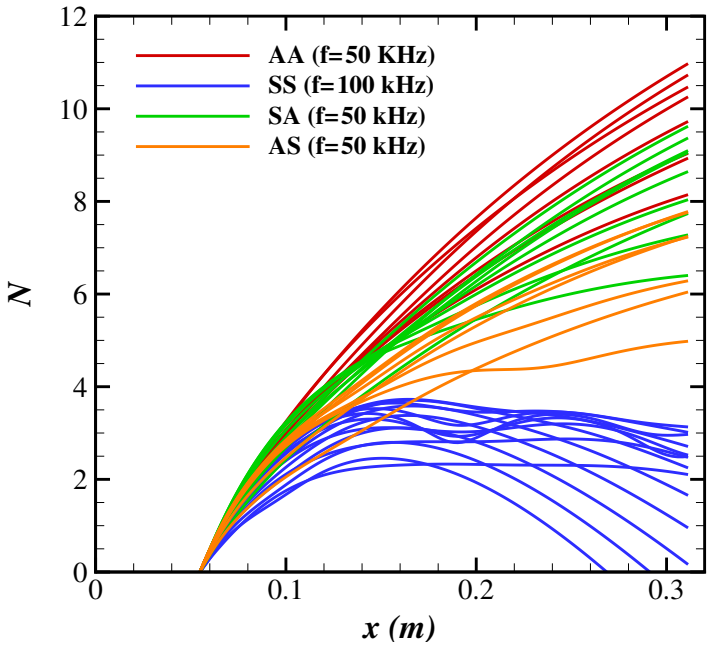

(b) $L_{w} / \lambda_{w}=4$

Figure 7. Effect of streamwise length of roughness patch with $k=272 \mu \mathrm{m}$ on $\mathrm{N}$-factor evolution for selected fixed-frequency disturbances from each mode type.

Figure 7(a) suggests that the instability amplification behind the shorter roughness patch $\left(L_{w} / \lambda_{w}=0.5\right)$ is rather modest, with a peak $\mathrm{N}$-factor of less than 5 , which is unlikely to produce transition onset over the plate even if the SLDT facility were to be operated in a noisy manner. On the other hand, Fig. 7(b) indicates that the peak N-factor in the $L_{w} / \lambda_{w}=4.0$ case approaches a value of $\mathrm{N}=11$. Therefore, if one assumes that the onset of transition correlates with $\mathrm{N}=10$ in a low disturbance environment analogous to SLDT, then transition onset should occur ahead of $x=0.3 \mathrm{~m}$ in this case even when the wind tunnel is operated in a quiet mode as planned, especially considering that the present analysis does not include any additional amplification within the region of the roughness patch. The most amplified disturbance in the $L_{w} / \lambda_{w}=4.0$ case corresponds to an AA mode. However, even SA modes can achieve an $\mathrm{N}$-factor approaching 10, making it possible that transition could occur due to either AA or SA modes, depending on their upstream history (i.e., receptivity characteristics and disturbance evolution across the region of the roughness patch). Detailed boundary layer measurements of the type reported in Ref. [8] would help ascertain the relative roles of these two modes during the transition process. Furthermore, it may be noted that the instability analysis presented in this paper corresponds to purely convective instability modes within the wake region downstream of the roughness patch. However, there is also a possibility of a global instability within the region of the roughness patch. Given the relatively modest roughness heights considered herein (and the low reverse flow velocities within the regions of separated flow), the likelihood of a global instability may not be large; however, we plan to perform DNS computations in the near future to characterize the instability evolution within the region of the roughness patch. 
Streamwise evolution of the $\left|u^{\prime}\right|$ mode shape for the most amplified disturbance from selected modal families for the roughness patch with $L_{w} / \lambda_{w}=0.5$ is shown in Figs. 8(a) and 8(b). The mean wake structure in this case is dominated by the centerline streak corresponding to the region of retarded flow behind the protuberances within the localized roughness patch. Accordingly, the strongest fluctuations occur in the vicinity of $z=0$. Both modes shown in Fig. 8 are antisymmetric about the centerplane, so that there are two peaks in the $\left|u^{\prime}\right|$ mode shape, one at either side of the center plane. As the streak becomes weaker toward the aft portion of the computational domain, the mode shape becomes less concentrated in the spanwise direction and secondary peaks near the lateral boundaries become more prominent with increasing $x$. Consistent with the symmetry properties of the AA and SA modes, these secondary peaks occur in pairs for the AA mode (one on either side of the $z / \lambda_{z}= \pm 0.5$ planes) whereas the SA mode exhibits a single additional peak at each lateral boundary. As a result of the progressive strengthening of this peak with increasing $x$, the peaks along $z / \lambda_{z}= \pm 0.5$ planes become the dominant peaks at the final station $(x=0.312 \mathrm{~m})$ as seen from Fig. 8(b).

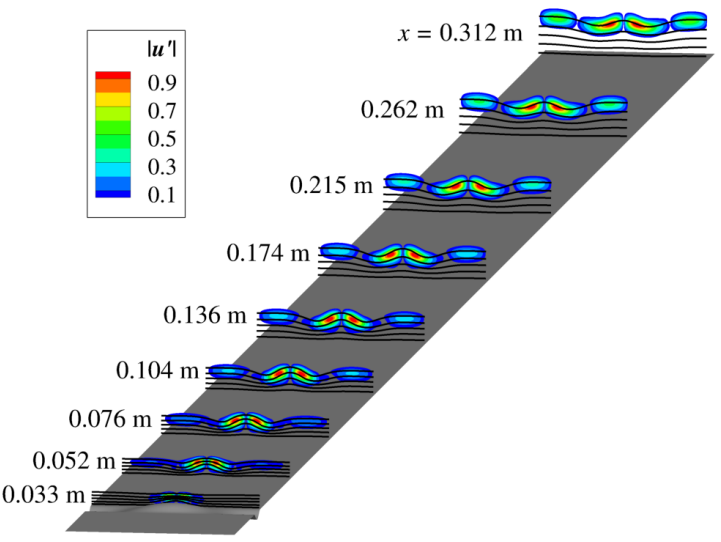

(a) AA mode

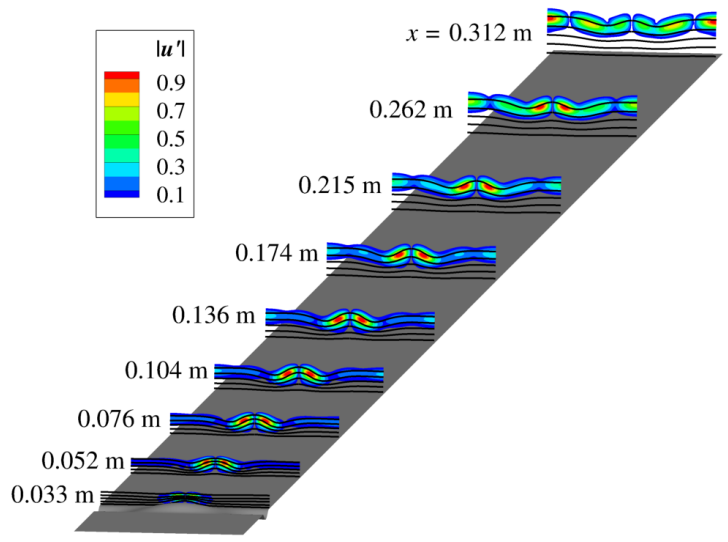

(b) SA mode

Figure 8. Axial variation in $\left|u^{\prime}\right|$ mode shapes for dominant AA mode with $f=60 \mathrm{kHz}$ from Fig. 7(a), i.e., for a roughness patch with $k=240 \mu \mathrm{m}$ and $L_{w} / \lambda_{w}=0.5$.

Mode shape evolution for the roughness patch with $L_{w} / \lambda_{w}=4.0$ is shown in Figs. 9(a) through 9(d), where a single dominant mode from of each of the AA, SA, AS, and SS mode types is included. As seen from Eq. (2), the roughness height distribution along the spanwise end planes $z / \lambda_{z}= \pm 0.5$ planes is equivalent to that along the center plane at $z / \lambda_{z}=0$, except for a phase difference of $\pi$ radians. Yet, because of the finite length of the roughness patch (containing just 4 wavelengths) and the relatively significant variations in the incoming boundary layer across a streamwise interval of $\lambda_{w} / 2$, the streak structure in the vicinity of the lateral end planes is similar, but not quite the same as that along the center plane, yielding a weakly modulated pattern of streaks along the spanwise direction. Effects of spanwise modulation of this type on the streak instabilities and the associated mode shapes have been studied in the context of crossflow instability evolution in the boundary layer flow over a swept wing ${ }^{34}$ and a yawed cone. $^{35}$ Not unlike the findings in those papers, the differences in boundary layers crests near the spanwise end planes and the centerplane are significant enough to yield highly visible differences in the spanwise structure of the $\left|u^{\prime}\right|$ mode shapes in those regions as seen from Fig. 9.

Figure 9(a) shows that the most amplified mode (mode AA) in the $L_{w} / \lambda_{w}=4.0$ case is primarily concentrated on either sides of the crest at $z / \lambda_{z}=0$, with secondary peaks that straddle the $z / \lambda_{z}= \pm 0.5$ planes. For $x \leq 0.099 \mathrm{~m}$, i.e., within the upstream portion of the roughness wake, there are tertiary peaks on either sides of the valley planes at $z / \lambda_{z}$ $= \pm 0.25$; however, these peaks are almost invisible on the scale of this plot and disappear as the wake amplitude becomes smaller at the downstream locations. Inspection of the instantaneous spanwise structure of the mode shape reveals that the AA mode corresponds to a fundamental, sinuous mode, whereas the SA mode corresponds to a subharmonic sinuous mode. Here, the classification between fundamental and subharmonic modes refers to spanwise wavelengths of the instability wave field with respect to the wavelength $\lambda_{z}$ of the roughness height distribution. According to this classification, the remaining two modes, i.e., modes AS and SS, correspond to subharmonic sinuous and fundamental varicose modes, respectively.

Fig. 9(d) shows that $u$ ' fluctuations associated with the SS mode (a fundamental varicose mode) are mainly concentrated within the central half of the streak, i.e, approximately between $z / \lambda_{z}= \pm 0.25$ and there are negligible 
fluctuations near the spanwise end planes within the upstream part of the wake region, i.e., $x \leq 0.156 \mathrm{~m}$. However, at downstream locations, the $u$ ' fluctuations progressively spread out in the spanwise direction. This suggests that, as the streak amplitude decreases in the downstream direction, the SS mode evolves into a first mode instability wave distorted by the streaks.

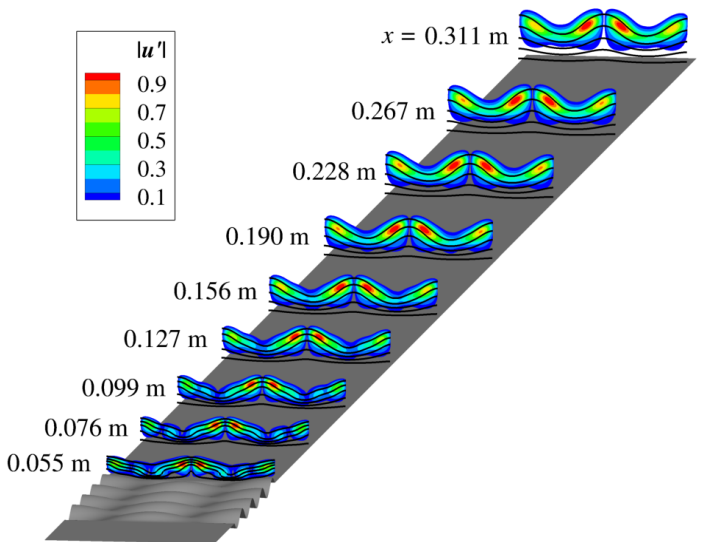

(a) AA mode $(f=50 \mathrm{kHz})$

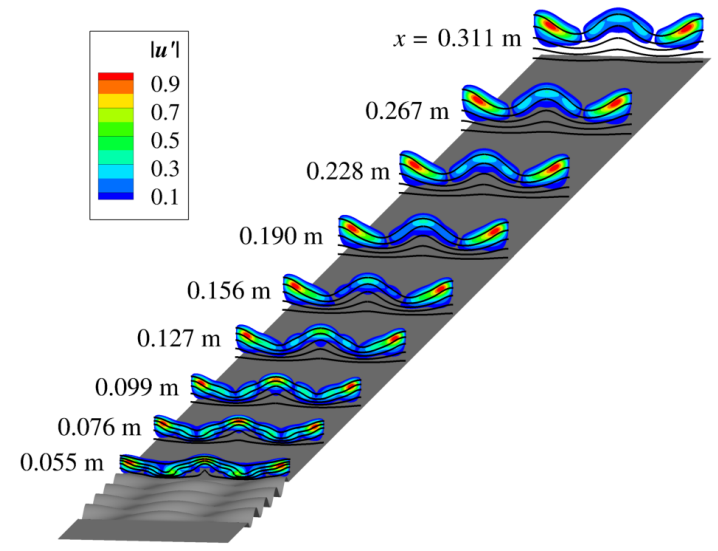

(c) AS mode $(f=50 \mathrm{kHz})$.

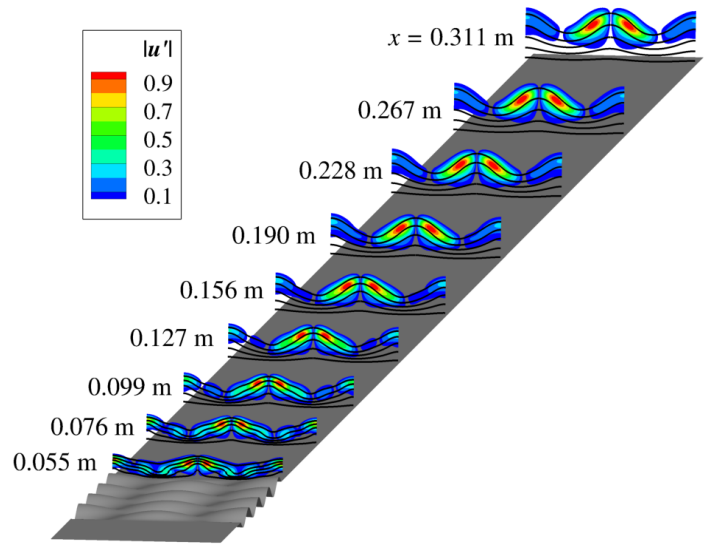

(b) SA mode $(f=50 \mathrm{kHz})$

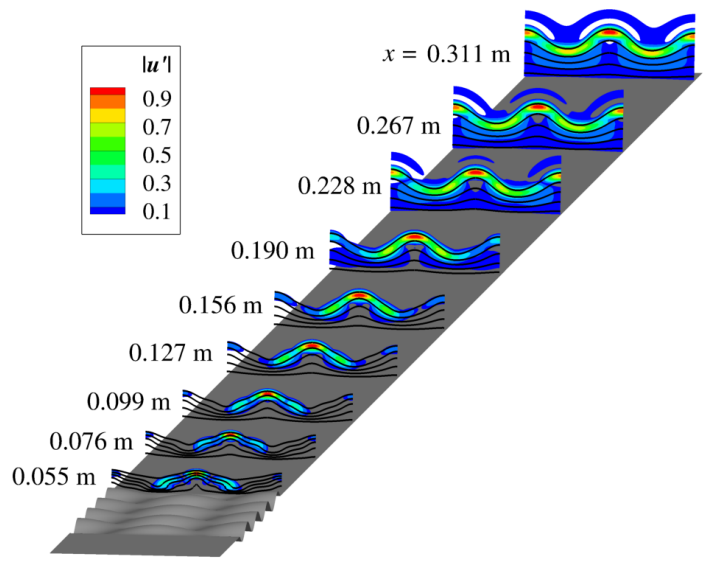

(d) SS mode $(f=100 \mathrm{kHz})$.

Figure 9. Mode shapes of $\left|\mathbf{u}^{\prime}\right|$ fluctuation associated with dominant instability modes from various families. Roughness patch parameters correspond to $k=240 \mu \mathrm{m}$ and $L_{w} / \lambda_{w}=4.0$. Line contours in black correspond to basic state $u$-velocity distribution across the wake.

\section{Summary and Concluding Remarks}

Numerical computations are performed to investigate the effects of extended surface waviness, or equivalently, a distributed region of smooth, densely packed roughness elements in both a Mach 5.3 axisymmetric boundary layer over a 7-deg. half-angle circular cone at high-altitude conditions and a Mach 3.5 flat plate boundary layer at the conditions of a planned experiment in the Supersonic Low Disturbance Tunnel at the NASA Langley Research Center. In the absence of the surface roughness, the Mach 5.3 boundary layer supports strong amplification of second mode (i.e., Mack mode) instability waves so that transition onset occurs near 83 percent of the body length. On the other hand, the Mach 3.5 boundary layer supports relatively weak amplification of first mode instabilities, and hence, transition is not expected to occur within the length of the plate. For peak roughness heights of 25 percent or less with respect to the boundary layer thickness, the time accurate computations for the Mach 5.3 configuration converge to a steady state, suggesting the flow to be globally stable in this case.

Analysis of the steady state solutions for both flow configurations (HIFiRE-1 cone and Mach 3.5 flat plate boundary layer) revealed that an extended patch of surface roughness that is comprised of multiple streamwise 
wavelengths corresponding to a regular pattern of distributed, smooth-shaped roughness elements (or, equivalently, surface waviness) induces very similar streamwise streaks as those observed in the previous studies for a single array of discrete roughness elements. Furthermore, a spanwise alignment of the protuberance elements within successive streamwise wavelengths of the roughness patch can lead to substantial reinforcement of the streak amplitudes associated with each localized segment of the overall distribution, similar to the previous findings for discrete roughness elements arranged in tandem. ${ }^{7,8}$ Computations also suggest that the higher streak amplitudes immediately behind a longer roughness patch may decay more slowly in comparison with the decay observed behind a shorter segment of the roughness distribution.

Convective instabilities in the wake of the roughness patch are examined, and their connection, where it exists, with the Mack mode instabilities in the unperturbed boundary layer over the HIFiRE-1 cone and first mode instabilities in the flat plate boundary layer is shown. For both flow configurations, there exist additional instabilities in the form of shear layer modes that owe their existence to the localized regions of high shear associated with the spanwise varying streaks. Not surprisingly, therefore, the overall amplification of these shearlayer modes, vis-à-vis the peak amplification of modes related to the Mack mode or first mode instabilities, is found to vary with the details of the roughness patch and the resulting amplitudes of the boundary layer streaks. Somewhat surprisingly, for the HIFiRE-1 configuration, a large patch of roughness corresponding to $L_{w} / \lambda_{w}=12$ (i.e., $L_{w} / \delta \approx 50$ ) and $k / \delta=0.2$ is found to result in a relatively small change in the $\mathrm{N}$-factor envelope (and hence, a correspondingly small delay in the estimated transition location), in spite of the strong distortion of the boundary layer flow due to the roughness patch.

Computations for the Mach 3.5 flat plate configuration illustrate the effects of roughness geometry on both mean wake characteristics and the modal instabilities of the wake flow. A comparison of streak structures within the wakes of two roughness patches with $\lambda_{w}=4.0, k=272 \mu \mathrm{m}$ (in relation to an approaching boundary layer thickness of slightly less than $0.6 \mathrm{~mm}$ ), and $L_{w} / \lambda_{w}=0.5$ and $L_{w} / \lambda_{w}=4.0$, respectively, underlined the cumulative effect of roughness length on the streak amplitude. Instability amplification behind the longer patch with $L_{w} / \lambda_{w}=4.0$ was found to be strong enough to potentially cause an earlier onset of transition at $x<0.3 \mathrm{~m}$, indicating an opposite effect of the roughness patch on transition in comparison with the Mach 5.3 flow configuration. The finding that a regular pattern of roughness elements can accelerate transition at supersonic Mach numbers, but delay it at higher Mach numbers has an experimental precedent in the wind tunnel investigations by Holloway and Sterrett as noted in the work of Paredes et al. ${ }^{28}$ Our currently ongoing work is focused on a more complete description of the wake instability characteristics as well as on the instabilities within the region of the roughness patch that were not examined herein, and on the comparison of predictions based on the stability theory with DNS simulations.

The computations presented in this paper share certain common features with the numerical simulations by Muppidi et al., ${ }^{13}$ such as the structure of the mean flow above the roughness patch and immediately behind it. However, one major difference between the two is related to the nature of the flow instabilities. Their computations revealed a sustained growth of unsteady disturbances without any external physical forcing, whereas the analysis presented in the current paper is focused on convective instabilities. Time accurate solutions for the roughness patch over the HIFiRE-1 cone relaxed to a steady state solution and the magnitude of reversed flow velocities in the vicinity of the roughness patches on both HIFiRE-1 and the flat plate configurations was relatively small. Further work is necessary to clarify this discrepancy, which will be investigated in our future computations.

\section{Acknowledgments}

This work was performed as part of the Transformational Tools and Technologies project of the NASA Transformative Aeronautics Concepts Program and the Hypersonic Technology Project. Computational resources for this work were provided by the NASA High-End Computing (HEC) Program through the NASA Advanced Supercomputing (NAS) Division at Ames Research Center. The authors would like to acknowledge useful technical discussions with Dr. Amanda Chou at NASA Langley Research Center. Comments on this paper by Dr. Balaji Venkatachari, Ms. Elizabeth Lee-Rausch, and Dr. Steve Bauer are also gratefully acknowledged.

\section{References}

1 Berry, S. A., Horvath, T. J., Greene, F. A., Kinder, G. R., and Wang, K. C., "Overview of Boundary Layer Transition Research in Support of Orbiter Return to Flight," AIAA Paper 2006-2918, June 2006.

2 Van Driest, E. R. and McCauley, W. D., "The Effect of Controlled Three-Dimensional Roughness on BoundaryLayer Transition at Supersonic Speeds," J. Aero. Sc., Vol. 27, No. 4, 1960, pp. 261-271.

3 Casper, K., Wheaton, B., Johnson, H., and Schneider, S., "Effect of Freestream Noise on Roughness-Induced Transition at Mach 6," AIAA Paper 2008-4291, 2008. 
4 Schneider, S. P., "Effects of Roughness on Hypersonic Boundary Layer Transition," Journal of Spacecraft and Rockets, Vol. 45, No. 2, 2008, pp. 193-209.

5 Schneider, S. P., "Hypersonic Boundary-Layer Transition on Blunt Bodies with Roughness," AIAA Paper 2008501, 2008.

6 Choudhari, M., Li, F., Paredes, P., and Chang, C.-L., "Boundary Layer Transition on Hypersonic Vehicles: Effects of Surface Roughness and Surface Blowing," Multiphysics Phenomena Analysis on Boundary Layer Stability in Hypersonic Regime - STO-AVT-289 Lecture Series, von Karman Institute for Fluid Dynamics, Sint-Genesius-Rode, Belgium, Sep. 18-20, 2017.

7 Choudhari, M., Li, F., Wu, M., Chang, C.-L., and Edwards, J. A., "Laminar Turbulent Transition behind Discrete Roughness Elements in a High-Speed Boundary-Layer,” AIAA Paper 2010-1575, 2010.

8 Chou, A. and Kegerise, M., "Transition Induced by a Streamwise Array of Roughness Elements on a Supersonic Flat Plate," AIAA Paper 2017-4515, 2017.

9 Reda, D. C., "Correlation of Nosetip Boundary-Layer Transition Data Measured in Ballistics-Range Experiments," AIAA Journal, Vol. 19, No. 3, 1981, pp. 329-339.

${ }^{10}$ Reshotko, E. and Tumin, A., "Role of Transient Growth in Roughness-Induced Transition," AIAA Journal, Vol. 42, No. 4, 2004, pp. 766-770.

${ }^{11}$ Reda, D. C., Wilder, M. C., Bogdanoff, D. W., and Prabhu, D. K., "Transition Experiments on Blunt Bodies with Distributed Roughness in Hypersonic Free Flight," Journal of Spacecraft and Rockets, Vol. 45, No. 2, MarchApril 2008, pp. 210-215.

${ }^{12}$ Hollis, B., "Correlation of Recent and Historical Rough-Wall Transition Data on Hemispherical Nosetips," AIAA Paper 2017-3986, 2017.

${ }^{13}$ Muppidi, S. and Mahesh, K., "Direct Numerical Simulations of Roughness-Induced Transition in Supersonic Boundary Layers," J. Fluid. Mech., Vol. 693, 2012, pp. 28-56.

${ }^{14}$ Kimmel, R. L., Adamczak, D. A., Paull, A., Paull, R., Shannon, J., Pietsch, R., Frost, M., Alesi, H., "HIFiRE-1 Ascent-Phase Boundary-Layer Transition," Journal of Spacecraft and Rockets, Vol. 52, 2015, pp. 217-230.

${ }^{15}$ Li, F., Choudhari, M., Chang, C.-L., Kimmel, R. L., Adamczak, D. A., and Smith, M., "Transition Analysis for the Ascent Phase of HIFiRE-1 Flight Experiment," Journal of Spacecraft and Rockets, Vol. 52, 2015, pp. 1283-1293.

${ }^{16} \mathrm{Wu}, \mathrm{M}$. and Martin, M. P., "Direct Numerical Simulation of Supersonic Boundary Layer over a Compression Ramp," AIAA Journal, Vol. 45, No. 4, 2007, pp. 879-889.

${ }^{17}$ Jiang, G. S. and Shu, C. W., "Efficient Implementation of Weighted ENO Schemes," J. Comp. Phys., Vol. 126, No. 1, 1996, pp. 202-228.

${ }^{18}$ Williamson, J., "Low-Storage Runge-Kutta Schemes," J. Comp. Phys., Vol. 35, No. 1, 1980, pp. 48-56.

${ }^{19}$ Duan, L., Beekman, I., and Martin, M. P., "Direct Numerical Simulation of Hypersonic Turbulent Boundary Layers. Part 3: Effect of Mach Number," J. Fluid. Mech., Vol. 672, 2011, pp. 245-267.

${ }^{20}$ Duan, L., Choudhari, M., and Li, F., "Direct Numerical Simulation of Crossflow-Induced Transition in a Swept Wing Boundary Layer," AIAA Paper 2013-2617, 2013.

${ }^{21}$ Choudhari, M., Li, F., Duan, L., Carpenter, M. H., Streett, C. L., and Malik, M. R., "Towards Bridging the Gaps in Holistic Transition Prediction via Numerical Simulations,” AIAA Paper 2013-2718, 2013.

${ }^{22}$ Choudhari, M., Li, F., Bynum, M. Kegerise, M. A., and King, R. A., "Computations of Disturbance Amplification Behind Isolated Roughness Elements and Comparison with Measurements," AIAA Paper 2015$2625,2015$.

${ }^{23}$ Wheaton, B. M., "Roughness-Induced Instabilities in a Mach-6 Laminar Boundary Layer," Ph.D. Thesis, Purdue Univ., School of Aeronautics and Astronautics, West Lafayette, IN, Dec. 2012.

${ }^{24}$ Li, F. and Choudhari, M., "Spatially Developing Secondary Instabilities and Attachment Line Instability in Supersonic Boundary Layers," AIAA Paper 2008-590, 2008.

${ }^{25} \mathrm{Li}$, F. and Choudhari, M., "Spatially Developing Secondary Instabilities in Compressible Swept Airfoil Boundary Layers," Theoretical and Computational Fluid Dynamics, Vol. 25, June 2011, pp. 65-84.

${ }^{26}$ Paredes, P., Choudhari, M., and Li, F., "Transition due to Streamwise Streaks in a Supersonic Flat Plate Boundary Layer," Phys. Rev. Fluids, Vol. 1, 2016, pp. 083601-1-23.

${ }^{27}$ Paredes, P., Choudhari, M., and Li, F., "Instability Wave-Streak Interactions in a Supersonic Boundary Layer," J. Fluid Mech., Vol. 831, 2017, pp. 524-553.

${ }^{28}$ Paredes, P., Choudhari, M., and Li, F., "Instability Wave-Streak Interactions in a Hypersonic Boundary Layer at Fight Conditions," J. Fluid Mech., 2018 (In review).

${ }^{29}$ vulcan-cfd.larc.nasa.gov (last accessed April 17, 2018). 
${ }^{30}$ van Albada, G. D., van Leer, B. and Roberts, W. W., "A Comparative Study of Computational Methods in Cosmic Gas Dynamics," Astronomy and Astrophysics, Vol. 108, 1982, pp. 76-84.

${ }^{31}$ Edwards, J. R., "A Low-Diffusion Flux-Splitting Scheme for Navier-Stokes Calculations," Computer and Fluids, Vol. 6, 1997, pp. 635-659.

${ }^{32}$ Litton, D., Edwards, J., and White, J., "Algorithmic Enhancements to the VULCAN Navier-Stokes Solver," AIAA Paper 2003-3979, 2003.

${ }^{33}$ Choudhari, M., Li, F., and Edwards, J. A., "Stability Analysis of Roughness Array Wake in a High-Speed Boundary Layer," AIAA Paper 2009-0170.

${ }^{34}$ Choudhari, M., Li, F., Paredes, P., "Influence of Stationary Crossflow Modulation on Secondary Instability," AIAA Paper 2016-3788, 2016.

${ }^{35}$ Choudhari, M., Li, F., Paredes, P., and Duan, L., "Nonlinear Evolution and Breakdown of Azimuthally Compact Crossflow Vortex Pattern over a Yawed Cone," AIAA Paper 2018-1823, 2018. 